\title{
Similarity Measures Based on q-Rung Linear Diophantine Fuzzy Sets and Their Application in Logistics and Supply Chain Management
}

\author{
Muhammad Qiyas $\mathbb{D}^{1},{ }^{1}$ Muhammad Naeem, ${ }^{2}$ Saleem Abdullah, ${ }^{1}$ Neelam Khan, \\ and Asad $\mathrm{Ali}^{3}$ \\ ${ }^{1}$ Department of Mathematics, Abdul Wali Khan University, Mardan, Pakistan \\ ${ }^{2}$ Deanship of Combined First Year, Umm Al-Qura University, Makkah, Saudi Arabia \\ ${ }^{3}$ Department of Mathematics, Hazara University, Mansehra, KP, Pakistan \\ Correspondence should be addressed to Muhammad Qiyas; muhammadqiyas@awkum.edu.pk
}

Received 18 August 2021; Revised 17 November 2021; Accepted 24 December 2021; Published 20 January 2022

Academic Editor: Musavarah Sarwar

Copyright (c) 2022 Muhammad Qiyas et al. This is an open access article distributed under the Creative Commons Attribution License, which permits unrestricted use, distribution, and reproduction in any medium, provided the original work is properly cited.

With the frequent occurrence of emergency events, decision-making (DM) plays an increasingly significant role in coping with them and has become an important and the challenging research focus recently. It is critical for decision makers to make accurate and reasonable emergency judgments in a short period as poor decisions can result in enormous economic losses and an unstable social order. As a consequence, this work offers a new DM approach based on novel distance and similarity measures using $q$-rung linear Diophantine fuzzy ( $q$-RLDF) information to assure that DM problems may be addressed successfully and fast. One of the useful methods for determining the degree of similarity between the objects is the similarity measure. In this paper, we propose some new $q$-rung linear Diophantine fuzzy ( $q$-ROLDF) distances and similarity measures. The Jaccard similarity measure, exponential similarity measure, and cosine and cotangent function-based similarity measures are proposed for $q$-LDFSs. The defined similarity measures are applied to the logistics and supply chain management problem, and the results are discussed. A comparison of new similarity measures is developed, and the proposed work's advantages are discussed.

\section{Introduction}

Models of knowledge-based decision-making attract substantial attention in industry and academia. A significant number of original research and thesis studies were undertaken to create effective decision support systems (DSSs) to promote managerial decision. DSS is classified as a specific class of electronic data system which supports decisionmaking (DM) management activities. In the early 1970s, the DSS idea was developed by Scott Morton's work. The methodology aims to evaluate strategic decisions in a complex and poorly organized environment, providing guidance to decision makers. DSSs have some huge benefits in decision-making by helping decision makers in their challenges and improve the effectiveness of the decisionmaking process [1].
For the purpose of logistics support, the better selection of logistic providers (LPs) is now important with the growth of the supply chain theories. Over the past decades, there has been a drastic change in the course of DSS [2]. Computer and technical experts have made great efforts to systematic processes of decision-making in the engineering and manufacturing industries [3]. Zha et al. [4], for example, developed a compromise decision-supporting issue approach and the fuzzy synthetic decision model (FSDM). Implementation of the DSS of multicriteria decision-making (MCDM) in supply chains is a constant challenge [5-7]. The reliability of the decision criteria in many decision-making problems (DMPs) is strictly dependent as external weights on the stakeholders and customer expectations. The delivery of decisions through solid and numerical values is difficult for clients. Fuzzy 
linguistic variables enable us to ensure judgmental consistency.

Zadeh [8] developed the concept of fuzzy set (FS) in 1965 , which addressed the membership degree (MD), and Atanassov [9] generalized the concept of FS by including nonmembership degree (NMD). IFS is a significant generalization of fuzzy sets that has been developed as a useful tool for understanding uncertain data. Pattern detection, decision-making, cluster analysis, and a variety of other areas are all derived from the IFS. In IFS theory, an element's $\mathrm{MD}$ and NMD must be between 0 and 1 , and the number of MD and NMD must be between 0 and 1 . As a result, in some practical applications, the values of MD and NMD of an element whose number of squares of MD and NMD may be greater than one are assigned to decision makers or decision experts. In such instances, the IFS has failed to clarify true or uncertain facts. Yager addressed this form of vague data by adding a new concept Pythagorean fuzzy set (PFS). Yager increased the space of MD and NMD by requiring that the square sum of MD and NMD lies between 0 and 1. PFSs [10] have found the complicated and indeterminacy problems in the assessment detail. Similar to the IFS, the PFS considered the function of MD $u$ and NMD $v$, but with the condition that the square sum of MD and NMD must lie between 0 and 1. This means that the PFs are more generalized and comprehensive than the IFS, making them more useful to decision makers. If an expert assigns MD 0.7 and NMD 0.6 to an object, this information does not satisfy the IFS condition, but it is clear that these data can only be conveyed using PFS. As a result, IF decision-making and SMs are distinct from PF decision-making and SMs. Finally, this shows that PFS can covenant more effectively than IFS. Many researchers and investigators have evolved various decision-making methods and techniques [11-15]. Verma [16] defined generalized SMs for PFSs and their applications to MADM. The extended version of the PF-TOPSIS method was studied and applied to MCDM by Zhang and Xu [17]. Peng and Yang [18] introduced the new operations of subtraction and division and studied their fundamental properties. Reformat and Yager built a recommender framework based on collaboration under PFS information in [19]. PFSs are studied by various authors, who introduced various models for group decision-making problems [20-23]. In [24-28], various aggregation operators based on Bonferroni mean (BM) operators, BM with geometric mean, and other aggregation operators were described. Du [29] defined Minkowski-type distance measures for generalized orthopair fuzzy sets. Ejegwa [30] developed a modified Zhang and Xu's distance measure for PFSs and its application to pattern recognition problems.

Due to the restrictions on the MD and NMD in the IFS and PFS, the information for an object does not clarify, and both concepts have failed to convey the information about an object in certain cases. As a result, in [31], Yager introduced a new concept called $q$-rung orthopair fuzzy set ( $q$ ROFS), which expands the MD and MND space by relaxing the condition to sum of the $q$ th power of MD $u$ and NMD $v$, i.e., $(u)^{q}+(v)^{q} \leq 1$, where $q$ is equal to or less than 1 . This means that if $q=1$, then $q$-ROFS will be reduced to the IFS, and if $q=2, q$-ROFS will be reduced to the PFS. Clearly, $q$ ROFS is a more generalized framework than the IFS and PFS for explaining unclear and ambiguous details. Liu and Wang described the algebraic sum and product operation for $q$ ROFSs in [32] and studied their application in MCDM. In [33], aggregation operators for $q$-ROFSs based on Maclaurin symmetric mean (MSM) were created. Heronian mean operator [34], partitioned Bonferroni mean operator [35], and power Bonferroni mean operator [36] were developed by the investigator and studied. Liu and $\mathrm{Xu}$ developed an MCDM technique for green supplier selection problems under $q$-ROFSs in [37].

The similarity measure (SM) is a powerful method for determining the degree of similarity and dissimilarity between two items in multicriteria decision-making (MCDM) theory and pattern recognition. For the last few decades, the theory and implementations of SMs have been studied. The authors of [38-41] predicted a wide range of SMs for two IFSs. Liu and Cheng's SMs were modified by Mitchel [42] and also applied to MCDM. Park et al. [43] evaluated the generalized form of fuzzy Hamming distance measure (HDsM), developed IFHDM, and proposed various SMs to apply to the MCDM problem. Torra and Narukawa created some new SMs based on Hausdorff distance and discussed some applications in [44]. Using geometric aggregation operators, Xia and $\mathrm{Xu}$ defined the concept of distance measure (DsM) and SM and applied them to the MCDM problem in [45]. Ye [46] developed IF cosine SMs using the cosine feature and applied them to the MCDM problem. Hung [47] specified the likelihood-based SM for the IFS and applied it to medical diagnosis. Shi and Ye provided a modified form of CSMs in [48]. Maoying defined the cotangent SM between two IFSs for MD [49]. Rajarajeswari and Uma described a modified version of cotangent SMs [50], in which they considered MD, NMD, and indeterminacy degrees designated in the IFS. Szmidt introduced the distance measure of IFSs and also developed SMs. In [51-54], some different and generalized distance measures and SMs of IFSs were found. $\mathrm{Li}$ and $\mathrm{Lu}$ [55] defined some novel similarity and distance measures of PFSs and their applications. Verma and Merigo [56] proposed generalized SMs for PFSs and their applications to MADM. Zeng et al. [57] proposed q-rung orthopair fuzzy weighted induced logarithmic distance measures and their application in MADM. Akram et al. [58] defined a novel MCGDM analysis under $m$-polar fuzzy soft expert sets. Akram et al. [59] proposed attribute reduction algorithms for $m$-polar fuzzy relation decision systems. Ali and Sarwar [60] developed a novel technique for group DM under the fuzzy parameterized-rung orthopair fuzzy soft expert framework. Ali and Akram [61] proposed a DM method based on fuzzy $N$-soft expert sets.

Wang et al. [62] defined the SMs for two q-ROFSs and applied them to pattern recognition and MCDM problems. Peng and Liu established distance, information, and similarity measures, as well as their relationships, in [63]. The Minkowski-type DM, which includes HM, ED, and Chebyshev DsM, was developed and debated in MCDM by Du [29]. Ali addressed two other methods in [64], developing 
the notions of possibility and confidence, as well as credibility and certainty, in $q$-ROFSs. The proposed work's key motivation is to analyze each extension of the fuzzy set (FS) in detail; in intuitionistic fuzzy sets, the two memberships explain the object's ambiguity, but the IFS fails to explain the described real-life problem. Consider a real-world problem where the MD and NMD values are greater than 0.5 , i.e., 0.6 and 0.7; then, $0.6+0.7>1$, in this case, using the Pythagorean fuzzy sets to describe the real-world problem. When the PFS fails to define some information in a real-world problem, it does not explain the unknown information. As a result, it is failing to clarify uncertainty by using the $q$-rung orthopair fuzzy sets. As a result, the definition of a linear Diophantine fuzzy set (LDFS) was created to cover the importance of membership and nonmembership functions. Raiz and Hashmi introduced the principle of the linear Diophantine fuzzy set (LDFS) in [65], and they demonstrated that it is more generalized than IFS, PFS, and $q$ ROFSs. The IFS, PFS, and $q$-ROFSs are less descriptive and efficient than the LDFS. The key benefit of the LDFS is the availability of comparable parameters (RPs). The MD and NMD have more space than the IFS, PFS, and $q$-ROFSs due to these RPs.

The RPs in the LDFS are constrained and bounded to a finite space, i.e., the number of RPs must be less than or equal to 1 . We work on distance and similarity measures of $q$-rung linear Diophantine fuzzy sets to extend the theory and applications of $q$-RLDFS. The distance measure for $q$ RLDFNs will be constructed, and their relations with the similarity measure will be studied. We continue to develop different types of similarity measures and define the Jaccard similarity measure (JMS) of the $q$-RLDFS, which tells us whether the information about the two $q$-RLDFSs is similar or different. For two $q$-RLDFSs, we also expand the JSM to weighted and generalized weighted JSM. Also, for two qRLDFSs, the exponential similarity measure (ESM) was developed and extended for weighted and generalized weighted ESM. The proposed similarity study's applications are discussed at the end of the article. The proposed similarity measure was used to solve the logistics and supply chain management problem. At the conclusion of the paper, we use the suggested similarity measures to solve a realworld problem of supply chain management. We solve the logistic provider supply chain management problem and show that the proposed SMs are superior to other SMs, currently in use.

The layout is structured as follows: some basic concepts of FS, IFS, $q$-ROFS, and LDFS are offered in Section 2. In Section 3, we define the novel distance measure of $q$-rung linear Diophantine fuzzy sets and the Hamming DM. In Section 4 , we introduce a similarity measure of $q$-rung linear Diophantine fuzzy sets and different types of similarity measure such as Jaccard SM and exponential SM. In Section 5 , we present the cosine similarity measure for $q$-RLDFSs and cotangent function similarity measure for $q$-RLDFSs. In Section 6, we address the decision frames, a case study for $q$ RLFSs, and a numerical example given to demonstrate the application of the proposed method by using the proposed algorithms. In Section 7, we discuss the comparison between the existing methods and proposed method. In Section 8, this work is eventually outlined.

\section{Basic Concepts}

The basic concept of fuzzy sets and their extensions are offered in the current section.

Definition 1 (see [8]). Let $X$ be an arbitrary nonempty set. A fuzzy set (FS) $L$ is defined as

$$
L=\left\{\left(x, u_{L}(x)\right) \mid x \in X\right\},
$$

where the function $u_{L}$ is a mapping from $X \longrightarrow[0,1]$, and for every $x \in X, 0 \leq u_{L}(x) \leq 1$, and the function $u_{L}(x)$ is said to be the MD of $x$ in $X$.

Definition 2 (see [9]). Let $X$ be an arbitrary nonempty set. An IFS $B$ in $X$ is defined as

$$
B=\left\{\left(x, u_{B}(x), v_{B}(x)\right) \mid x \in X\right\},
$$

where $u_{B} \longrightarrow[0,1]$ and $v_{B} \longrightarrow[0,1]$ are called, respectively, MD and NMD functions such that $\forall x \in X: 0 \leq$ $u_{B}(x)+v_{B}(x) \leq 1$. Indeterminacy degree can be defined as

$$
\pi_{B}(x)=1-u_{B}(x)-v_{B}(x), \forall x \in X .
$$

Definition 3 (see [10]). Let $X$ be an arbitrary nonempty set. A $q$-ROFS is denoted by $B_{P}$ and mathematically defined as

$$
B=\left\{\left(x, u_{B}(x), v_{B}(x)\right) \mid x \in X\right\},
$$

where $u_{B}(x)$ and $v_{B}(x)$ are $\mathrm{MD}$ and NMD functions $: X \longrightarrow[0,1]$ with subject to $\left(u_{B}(x)^{q)}+\left(v_{B}(x)^{q)} \leq 1\right.\right.$. The hesitancy MD is denoted by

$$
\pi_{B}(x)=\sqrt{1-\left(u_{B}(x)^{q)}-\left(v_{B}(x)^{q)}\right.\right.} .
$$

$q$-ROFSs also have certain restrictions on $\mathrm{MDs}$ and NMDs.

Definition 4 (see [65]). Let $X$ be an arbitrary nonempty set. A LDFS is denoted by $G_{D}$ and mathematically defined as

$$
\left.G=\left\{x,\left\langle u_{G}(x), v_{G}(x)\right\rangle,\langle a, b\rangle\right): x \in X\right\},
$$

where $u_{G}(x), v_{G}(x), a, b \in[0,1]$ are $\mathrm{MD}, \mathrm{NMD}$, and RPs, respectively, and satisfy the condition $0 \leq a u_{G}(x)+$ $b v_{G}(x) \leq 1, \forall x \in X$ with $0 \leq a+b \leq 1$. The following is the hesitancy degree:

$$
\pi_{G}(x)=1-(a) u_{G}(x)-(b) v_{G}(x) .
$$

Definition 5 (see [66]). Let $X$ be an arbitrary nonempty set. Then, the $q$-rung linear Diophantine fuzzy set ( $q$-RLDFS) is represented by $T$ and mathematically defined as

$$
\left.T=\left\{x,\left\langle u_{T}(x), v_{T}(x)\right\rangle,\langle a, b\rangle\right): x \in X\right\},
$$


where $u_{T}, v_{T}, a, b \in[0,1]$ are $\mathrm{MD}, \mathrm{NMD}$, and reference parameters (RPs), respectively. These functions fulfill the restriction $0 \leq(a)^{q} u_{T}+(b)^{q} v_{T} \leq 1, \forall x \in X, q \geqslant 1$, with $0 \leq a^{q}$ $+b^{q} \leq 1, q \geq 1$. The percentage of hesitation can be measured as follows:

$$
\pi_{T}=\sqrt[q]{1-\left(\left(a^{q)} u_{T}(x)+\left(b^{q)} v_{T}(x)\right)\right.\right.}
$$

and $q$-rung linear Diophantine fuzzy number ( $q$-RLDFN) is defined as

$$
T=\left\{\left\langle u_{T}, v_{T}\right\rangle,\langle a, b\rangle\right\}
$$

\section{Distance Measure of $q$-Rung Linear Diophantine Fuzzy Sets}

In this section, we describe the distance measure (DsM) between two $q$-RLDFSs, as well as their basic properties and their relation.

Definition 6. Consider a family of $q$-RLDFSs. Then, a mapping $d\left(\Upsilon_{1}, \Upsilon_{2}\right): q-R L D F(\Upsilon) \times q-R L D F(\Upsilon) \longrightarrow$ $[0,1]$ is said to be distance measure $(D M)$, where $\Upsilon_{1}, \Upsilon_{2}$, $\Upsilon_{3} \in q-R L D F(\Upsilon)$, if the following conditions hold:

(1) $0 \leq d\left(\Upsilon_{1}, \Upsilon_{2}\right) \leq 1$.

(2) $d\left(\Upsilon_{1}, \Upsilon_{2}\right) \leq d\left(\Upsilon_{2}, \Upsilon_{1}\right)$.

(3) $d\left(\Upsilon_{1}, \Upsilon_{2}\right)=1 \Leftrightarrow \Upsilon_{1}=\Upsilon_{2}$.

(4) $d\left(\Upsilon_{1}, \Upsilon_{2}\right)^{c}=1 \Leftrightarrow \Upsilon_{1}$ is a crisp set.

(5) Let $\Upsilon_{1} \subseteq \Upsilon_{2} \subseteq \Upsilon_{3}$. Then, $d\left(\Upsilon_{1}, \Upsilon_{3}\right) \leq d\left(\Upsilon_{1}, \Upsilon_{2}\right)+$ $d\left(\Upsilon_{2}, \Upsilon_{3}\right)$.

Now, we define normalized Hamming distance and normalized Euclidean distance measure between two $q$ RLDFSs $\Upsilon_{1}$ and $\Upsilon_{2}$ in the following.

Definition 7. Consider two nonempty $q$-RLDFNs $\Upsilon_{1}=$ $\left(\left\langle u_{\Upsilon_{1}}\left(x_{i}\right), v_{\Upsilon_{1}}\left(x_{i}\right)\right\rangle,\left\langle a_{i}, b_{i}\right\rangle\right)$ and $\Upsilon_{2}=\left(\left\langle u_{\Upsilon_{2}}\left(x_{i}\right), v_{\Upsilon_{2}}\left(x_{i}\right)\right\rangle\right.$, $\left.\left\langle c_{i}, d_{i}\right\rangle\right)$ on the discrete nonempty set $\wp=\left(\rho_{1}, \rho_{2}, \ldots, \rho_{n}\right)$. Then, the normalized Hamming distance measure is denoted by $\operatorname{NHD}\left(\Upsilon_{1}, \Upsilon_{2}\right)$ and given as

$$
\operatorname{NHD}\left(\Upsilon_{1}, \Upsilon_{2}\right)=\frac{1}{4 n} \sum_{i=1}^{n}\left\{\begin{array}{c}
\left|u_{\Upsilon_{1}}^{q}\left(x_{i}\right)-u_{\Upsilon_{2}}^{q}\left(x_{i}\right)\right| \\
+\left|v_{\Upsilon_{1}}^{q}\left(x_{i}\right)-v_{\Upsilon_{2}}^{q}\left(x_{i}\right)\right| \\
+\left|a_{i}^{q}-c_{i}^{q}\right|+\left|b_{i}^{q}-d_{i}^{q}\right|
\end{array}\right\} .
$$

Definition 8. Consider two nonempty $q$-RLDFNs $\Upsilon_{1}=$ $\left(\left\langle u_{\Upsilon_{1}}\left(x_{i}\right), v_{\Upsilon_{1}}\left(x_{i}\right)\right\rangle,\left\langle a_{i}, b_{i}\right\rangle\right)$ and $\Upsilon_{2}=\left(\left\langle u_{\Upsilon_{2}}\left(x_{i}\right), v_{\Upsilon_{2}}\left(x_{i}\right)\right\rangle\right.$, $\left.\left\langle c_{i}, d_{i}\right\rangle\right)$ on the discrete nonempty set $\wp=\left(\rho_{1}, \rho_{2}, \ldots, \rho_{n}\right)$. Then, the normalized Euclidean distance is denoted by $\operatorname{NED}\left(\Upsilon_{1}, \Upsilon_{2}\right)$ and given as

$$
\operatorname{NED}\left(\Upsilon_{1}, \Upsilon_{2}\right)=\sqrt{\frac{1}{4 n} \sum_{i=1}^{n}}\left\{\begin{array}{c}
\left(u_{\Upsilon_{1}}^{q}\left(x_{i}\right)-u_{\Upsilon_{2}}^{q}\left(x_{i}\right)\right)^{2} \\
+\left(v_{\Upsilon_{1}}^{q}\left(x_{i}\right)-v_{\Upsilon_{2}}^{q}\left(x_{i}\right)\right)^{2} \\
+\left(a_{i}^{q}-c_{i}^{q}\right)^{2}+\left(b_{i}^{q}-d_{i}^{q}\right)^{2}
\end{array}\right\} .
$$

We further generalize the HDM and EDM for two $q$ RLDFSs $\Upsilon_{1}$ and $\Upsilon_{2}$ using a parameter $\lambda \geq 1$ into equations (13) and (14), respectively. The generalized distance measure (GDsM) of two $q$-RLDFSs $\Upsilon_{1}$ and $\Upsilon_{2}$ is given as

$\operatorname{GDM}\left(\Upsilon_{1}, \Upsilon_{2}\right)=\left\{\frac{1}{4 n} \sum_{i=1}^{n}\left(\begin{array}{c}\left|u_{\Upsilon_{1}}^{q}\left(x_{i}\right)-u_{\Upsilon_{2}}^{q}\left(x_{i}\right)\right|^{\lambda} \\ +\left|v_{\Upsilon_{1}}^{q}\left(x_{i}\right)-v_{\Upsilon_{2}}^{q}\left(x_{i}\right)\right|^{\lambda} \\ +\left|a_{i}^{q}-c_{i}^{q}\right|^{\lambda}+\left|b_{i}^{q}-d_{i}^{q}\right|^{\lambda}\end{array}\right)\right\}^{1 / \lambda}$.

Remark 1. If we put $\lambda=1$, then $\operatorname{GDM}\left(\Upsilon_{1}, \Upsilon_{2}\right)$ is reduced to $\operatorname{HD}\left(\Upsilon_{1}, \Upsilon_{2}\right)$.

Remark 2. If we put $\lambda=2$, then $\operatorname{GDM}\left(\Upsilon_{1}, \Upsilon_{2}\right)$ is reduced to $\operatorname{HD}\left(\Upsilon_{1}, \Upsilon_{2}\right)$.

Next, we use the weight of each $x_{i}$ with the weight vector $w=\left(w_{1}, \ldots, w_{n}\right)$ subject to $w_{i} \in[0,1]$ such that $\sum_{i=1}^{n} w_{i}=1$. Then, we propose a new DsM, so called the generalized weighted distance measure (GWDsM) of $\Upsilon_{1}$ and $\Upsilon_{2}$ which is denoted by $\operatorname{GWDM}\left(\Upsilon_{1}, \Upsilon_{2}\right)$ and defined as

$\operatorname{GWDM}\left(\Upsilon_{1}, \Upsilon_{2}\right)=\left\{\sum_{i=1}^{n} w_{i}\left(\begin{array}{c}\left|u_{\Upsilon_{1}}^{q}\left(x_{i}\right)-u_{\Upsilon_{2}}^{q}\left(x_{i}\right)\right|^{\lambda} \\ +\left|v_{\Upsilon_{1}}^{q}\left(x_{i}\right)-v_{\Upsilon_{2}}^{q}\left(x_{i}\right)\right|^{\lambda} \\ +\left|a_{i}^{q}-c_{i}^{q}\right|^{\lambda}+\left|b_{i}^{q}-d_{i}^{q}\right|^{\lambda}\end{array}\right)\right\}^{1 / \lambda}$.

\section{Similarity Measure of $q$-Rung Linear Diophantine Fuzzy Sets}

In this section, we define different types of similarity measure (SM) of $q$-RLDFS and also discuss the basic properties of the proposed SMs. We will use the notion of the Jaccard, exponential, cosine, and cotangent functions and develop the similarity measures between $q$-RLDFSs.

First, we give the definition of similarity measures between two $q$-RLDFSs.

Definition 9. Consider a family of $q$-RLDFNs. Then, a mapping $\operatorname{SM}\left(\Upsilon_{1}, \Upsilon_{2}\right): q-\operatorname{RLDF}(\Upsilon) \times q-\operatorname{RLDF}(\Upsilon) \longrightarrow$ 
$[0,1]$ is said to be a similarity measure $(\mathrm{SM})$, where $\Upsilon_{1}, \Upsilon_{2}, \Upsilon_{3} \in q-\operatorname{RLDF}(\Upsilon)$, if the following conditions hold:

(1) $0 \leq \operatorname{SM}\left(\Upsilon_{1}, \Upsilon_{2}\right) \leq 1$

(2) $\operatorname{SM}\left(\Upsilon_{1}, \Upsilon_{2}\right) \leq \operatorname{SM}\left(\Upsilon_{2}, \Upsilon_{1}\right)$.

(3) $\operatorname{SM}\left(\Upsilon_{1}, \Upsilon_{2}\right)=1 \Leftrightarrow \Upsilon_{1}=\Upsilon_{2}$.

(4) $\operatorname{SM}\left(\Upsilon_{1}, \Upsilon_{2}\right)^{c}=1 \Leftrightarrow \Upsilon_{1}$ is a crisp set.

(5) Let $\Upsilon_{1} \subseteq \Upsilon_{2} \subseteq \Upsilon_{3}$. Then, $\operatorname{SM}\left(\Upsilon_{1}, \Upsilon_{3}\right) \leq \min \left\{\operatorname{SM}\left(\Upsilon_{1}\right.\right.$, $\left.\left.\Upsilon_{2}\right)+\operatorname{SM}\left(\Upsilon_{2}, \Upsilon_{3}\right)\right\}$.

4.1. Jaccard Similarity Measure of $q-R L D F S$ s. This section consists of a novel SM between $q$-RLDFSs which is called the Jaccard similarity measure (JSM) between two $q$-RLDFSs. The JSM tells us the information whether the two $q$-RLDFSs are similar or distinct. The JSM provides information of similarity from 0 to 1 for the two $q$-RLDFSs. If the information of similarity of two $q$-RLDFSs is near to 1 , then both are similar to each other, and if the information of similarity is near to close to 0 , then both are dissimilar from each other. The JSM is very informative for similarity and dissimilarity for $q$-RLDFSs, so the JSM is useful in the decision-making problem and pattern recognition.

(1) $0 \leq \operatorname{JSM}\left(\Upsilon_{1}, \Upsilon_{2}\right) \leq 1$

(2) $\operatorname{JSM}\left(\Upsilon_{1}, \Upsilon_{2}\right)=\operatorname{JSM}\left(\Upsilon_{2}, \Upsilon_{1}\right)$

(3) $\operatorname{JSM}\left(\Upsilon_{1}, \Upsilon_{2}\right)=1$ iff $\Upsilon_{1}=\Upsilon_{2}$

Definition 10. Consider two nonempty $q$-RLDFNs $\Upsilon_{1}=$ $\left(\left\langle u_{\Upsilon_{1}}\left(x_{i}\right), v_{\Upsilon_{1}}\left(x_{i}\right)\right\rangle,\left\langle a_{i}, b_{i}\right\rangle\right)$ and $\Upsilon_{2}=\left(\left\langle u_{\Upsilon_{2}}\left(x_{i}\right), v_{\Upsilon_{2}}\left(x_{i}\right)\right\rangle\right.$, $\left.\left\langle c_{i}, d_{i}\right\rangle\right)$ on the discrete nonempty set $\wp=\left(\rho_{1}, \rho_{2}, \ldots, \rho_{n}\right)$. Then, the Jaccard similarity measure (JSM) is denoted by $\operatorname{JSM}\left(\Upsilon_{1}, \Upsilon_{2}\right)$ and given as

$$
\begin{aligned}
\operatorname{JSM}\left(\Upsilon_{1}, \Upsilon_{2}\right)=\frac{1}{n} \sum_{i=1}^{n} & \frac{u_{\Upsilon_{1}}^{q}(x) u_{\Upsilon_{2}}^{q}\left(x_{i}\right)+v_{\Upsilon_{1}}^{q}\left(x_{i}\right) v_{\Upsilon_{2}}^{q}\left(x_{i}\right)+a_{i}^{q} c_{i}^{q}+b_{i}^{q} d_{i}^{q}}{\left[\left(u_{\Upsilon_{1}}^{q}\left(x_{i}\right)\right)^{2}+\left(v_{\Upsilon_{1}}^{q}\left(x_{i}\right)\right)^{2}+\left(a_{i}^{q}\right)^{2}+\left(c_{i}^{q}\right)^{2}\right]} \\
& +\left[\left(u_{\Upsilon_{2}}^{q}\left(x_{i}\right)\right)^{2}+\left(v_{\Upsilon_{2}}^{q}\left(x_{i}\right)\right)^{2}+\left(b_{i}^{q}\right)^{2}+\left(d_{i}^{q}\right)^{2}\right] \\
& -\left[u_{\Upsilon_{1}}^{q}\left(x_{i}\right) u_{\Upsilon_{2}}^{q}\left(x_{i}\right)+v_{\Upsilon_{1}}^{q}\left(x_{i}\right) v_{\Upsilon_{2}}^{q}\left(x_{i}\right)+a_{i}^{q} c_{i}^{q}+b_{i}^{q} d_{i}^{q}\right]
\end{aligned}
$$

Theorem 1. Consider two nonempty q-RLDFNs $\Upsilon_{1}=\left(\left\langle u_{\Upsilon_{1}}\right.\right.$ $\left.\left.\left(x_{i}\right), v_{\Upsilon_{1}}\left(x_{i}\right)\right\rangle,\left\langle a_{i}, b_{i}\right\rangle\right)$ and $\Upsilon_{2}=\left(\left\langle u_{\Upsilon_{2}}\left(x_{i}\right), v_{\Upsilon_{2}}\left(x_{i}\right)\right\rangle,\left\langle c_{i}\right.\right.$, $\left.\left.d_{i}\right\rangle\right)$ on the discrete nonempty set $\wp=\left(\rho_{1}, \rho_{2}, \ldots, \rho_{n}\right)$. Then, $\operatorname{JSM}\left(\Upsilon_{1}, \Upsilon_{2}\right)$ defined in equation (11) satisfies the following conditions:

\section{Proof}

(1) As $\Upsilon_{1}, \Upsilon_{2}$ are two $q$-RLDFNs and we know that $x^{2}+y^{2} \geq 2 x y$,

$u_{\Upsilon_{1}}^{q}\left(x_{i}\right) u_{\Upsilon_{2}}^{q}\left(x_{i}\right)+v_{\Upsilon_{1}}^{q}\left(x_{i}\right) v_{\Upsilon_{2}}^{q}\left(x_{i}\right)+a_{i}^{q} c_{i}^{q}+b_{i}^{q} d_{i}^{q} \geq 0$,

$$
\left\{\begin{array}{c}
{\left[\left(u_{\Upsilon_{1}}^{q}\left(x_{i}\right)\right)^{2}+\left(v_{\Upsilon_{1}}^{q}\left(x_{i}\right)\right)^{2}+\left(a_{i}^{q}\right)^{2}+\left(c_{i}^{q}\right)^{2}\right]} \\
+\left[\left(u_{\Upsilon_{2}}^{q}\left(x_{i}\right)\right)^{2}+\left(v_{\Upsilon_{2}}^{q}\left(x_{i}\right)\right)^{2}+\left(b_{i}^{q}\right)^{2}+\left(d_{i}^{q}\right)^{2}\right] \\
-\left[u_{\Upsilon_{1}}^{q}\left(x_{i}\right) u_{\Upsilon_{2}}^{q}\left(x_{i}\right)+v_{\Upsilon_{1}}^{q}\left(x_{i}\right) v_{\Upsilon_{2}}^{q}\left(x_{i}\right)+a_{i}^{q} c_{i}^{q}+b_{i}^{q} d_{i}^{q}\right]
\end{array}\right\} \geq 0 .
$$

Thus, $J S M\left(\Upsilon_{1}, \Upsilon_{2}\right) \geq 0$, and summing up to $n$ and normalizing, we have $\operatorname{JSM}\left(\Upsilon_{1}, \Upsilon_{2}\right) \leq 1$. Hence, $0 \leq$ $\operatorname{JSM}\left(\Upsilon_{1}, \Upsilon_{2}\right) \leq 1$.

(2) The proof of $\operatorname{JSM}\left(\Upsilon_{1}, \Upsilon_{2}\right)=\operatorname{JSM}\left(\Upsilon_{2}, \Upsilon_{1}\right)$ is trivial.

(3) Suppose that $\Upsilon_{1}=\Upsilon_{2}$; then, $u_{\Upsilon}^{q}\left(x_{i}\right)=u_{\Upsilon_{2}}^{q}\left(x_{i}\right), v_{\Upsilon}^{q}$ $\left(x_{i}\right)=v_{\Upsilon_{2}}^{q}\left(x_{i}\right), a_{i}^{q}=c_{i}^{q}, b_{i}^{q}=d_{i}^{q}$. Then, equation (11) becomes

and

$$
\begin{aligned}
\operatorname{JSM}\left(\Upsilon_{1}, \Upsilon_{2}\right)=\frac{1}{n} \sum_{i=1}^{n} & \frac{u_{\Upsilon_{1}}^{q}(x) u_{\Upsilon_{1}}^{q}\left(x_{i}\right)+v_{\Upsilon_{1}}^{q}\left(x_{i}\right) v_{\Upsilon_{1}}^{q}\left(x_{i}\right)+a_{i}^{q} a_{i}^{q}+b_{i}^{q} b_{i}^{q}}{\left[\left(u_{\Upsilon_{1}}^{q}\left(x_{i}\right)\right)^{2}+\left(v_{\Upsilon_{1}}^{q}\left(x_{i}\right)\right)^{2}+\left(a_{i}^{q}\right)^{2}+\left(a_{i}^{q}\right)^{2}\right]} \\
& +\left[\left(u_{\Upsilon_{1}}^{q}\left(x_{i}\right)\right)^{2}+\left(v_{\Upsilon_{1}}^{q}\left(x_{i}\right)\right)^{2}+\left(b_{i}^{q}\right)^{2}+\left(b_{i}^{q}\right)^{2}\right] \\
& -\left[u_{\Upsilon_{1}}^{q}\left(x_{i}\right) u_{\Upsilon_{1}}^{q}\left(x_{i}\right)+v_{\Upsilon_{1}}^{q}\left(x_{i}\right) v_{\Upsilon_{1}}^{q}\left(x_{i}\right)+a_{i}^{q} a_{i}^{q}+b_{i}^{q} b_{i}^{q}\right]
\end{aligned}
$$




$$
\begin{aligned}
& =\frac{1}{n} \sum_{i=1}^{n} \frac{\left(u_{\Upsilon_{1}}^{q}(x)\right)^{2}+\left(v_{\Upsilon_{1}}^{q}\left(x_{i}\right)\right)^{2}+\left(a_{i}^{q}\right)^{2}+\left(b_{i}^{q}\right)^{2}}{\left[\begin{array}{c}
\left(u_{\Upsilon_{1}}^{q}\left(x_{i}\right)\right)^{2}+\left(v_{\Upsilon_{1}}^{q}\left(x_{i}\right)\right)^{2} \\
+\left(a_{i}^{q}\right)^{2}+\left(a_{i}^{q}\right)^{2}
\end{array}\right]} \\
& +\left[\left(u_{\Upsilon_{1}}^{q}\left(x_{i}\right)\right)^{2}+\left(v_{\Upsilon_{1}}^{q}\left(x_{i}\right)\right)^{2}+\left(b_{i}^{q}\right)^{2}+\left(b_{i}^{q}\right)^{2}\right] \\
& -\left[\left(u_{\Upsilon_{1}}^{q}\left(x_{i}\right)\right)^{2}+\left(v_{\Upsilon_{1}}^{q}\left(x_{i}\right)\right)^{2}+\left(a_{i}^{q}\right)^{2}+\left(b_{i}^{q}\right)^{2}\right] \\
& =\frac{1}{n} \sum_{i=1}^{n} \frac{\left(u_{\Upsilon_{1}}^{q}(x)\right)^{2}+\left(v_{\Upsilon_{1}}^{q}\left(x_{i}\right)\right)^{2}+\left(a_{i}^{q}\right)^{2}+\left(b_{i}^{q}\right)^{2}}{\left[\left(u_{\Upsilon_{1}}^{q}\left(x_{i}\right)\right)^{2}+\left(v_{\Upsilon_{1}}^{q}\left(x_{i}\right)\right)^{2}+\left(a_{i}^{q}\right)^{2}+\left(a_{i}^{q}\right)^{2}\right]} \\
& =1 \text {. }
\end{aligned}
$$

Conversely, the proof is easy.

Next, we use the weight of each $x_{i}$ with the weight vector $w=\left(w_{1}, w_{2}, \ldots, w_{n}\right)^{T}$ subject to $w_{i} \in[0,1]$ such that $\sum_{i=1}^{n} w_{i}=1$. Then, we propose a new SM, called weighted Jaccard similarity measure of $\Upsilon_{1}$ and $\Upsilon_{2}$ which is denoted by $\operatorname{JSM}\left(\Upsilon_{1}, \Upsilon_{2}\right)$ and defined in the following equation:

$$
\begin{aligned}
\operatorname{WJSM}\left(\Upsilon_{1}, \Upsilon_{2}\right)=\sum_{i=1}^{n} w_{i} \frac{u_{\Upsilon_{1}}^{q}(x) u_{\Upsilon_{2}}^{q}\left(x_{i}\right)+v_{\Upsilon_{1}}^{q}\left(x_{i}\right) v_{\Upsilon_{2}}^{q}\left(x_{i}\right)+a_{i}^{q} c_{i}^{q}+b_{i}^{q} d_{i}^{q}}{\left[\left(u_{\Upsilon_{1}}^{q}\left(x_{i}\right)\right)^{2}+\left(v_{\Upsilon_{1}}^{q}\left(x_{i}\right)\right)^{2}+\left(a_{i}^{q}\right)^{2}+\left(c_{i}^{q}\right)^{2}\right]} . \\
+\left[\left(u_{\Upsilon_{2}}^{q}\left(x_{i}\right)\right)^{2}+\left(v_{\Upsilon_{2}}^{q}\left(x_{i}\right)\right)^{2}+\left(b_{i}^{q}\right)^{2}+\left(d_{i}^{q}\right)^{2}\right] \\
-\left[u_{\Upsilon_{1}}^{q}\left(x_{i}\right) u_{\Upsilon_{2}}^{q}\left(x_{i}\right)+v_{\Upsilon_{1}}^{q}\left(x_{i}\right) v_{\Upsilon_{2}}^{q}\left(x_{i}\right)+a_{i}^{q} c_{i}^{q}+b_{i}^{q} d_{i}^{q}\right]
\end{aligned}
$$

The proposed WJSM $\left(\Upsilon_{1}, \Upsilon_{2}\right)$ also satisfies the conditions of Theorem 1 .

4.2. Exponential Similarity Measure of $q$-RLDFSs. In this section, the exponential function with Euclidean and Hamming distances between two $q$-RLDFSs $\Upsilon_{1}$ and $\Upsilon_{2}$ is used to provide a new similarity measure between the two $q$ RLDFSs. Shepard predicted a universal law regarding distance and similarity measures in 1987. Both are related to an exponential function, and using the similarity measure based on the expansion function, the exponential distance measure is defined as

$$
\operatorname{ESM}_{d}\left(\Upsilon_{1}, \Upsilon_{2}\right)=E^{-d\left(\Upsilon_{1}, \Upsilon_{2}\right)}
$$

Now, we will talk about the properties of the distance measure, also known as axioms. Every distance measure must satisfy the following axioms:

(1) Equality: $d\left(\Upsilon_{1}, \Upsilon_{2}\right)=d\left(\Upsilon_{2}, \Upsilon_{1}\right)$ for every $\Upsilon_{1}$ and $\Upsilon_{2}$. Thus, $\operatorname{ESM}_{d}\left(\Upsilon_{1}, \Upsilon_{2}\right)=\operatorname{ESM}_{d}\left(\Upsilon_{2}, \Upsilon_{1}\right)$ for every $\Upsilon_{1}$ and $\Upsilon_{2}$.

(2) Minimality: $d\left(\Upsilon_{1}, \Upsilon_{2}\right)>d\left(\Upsilon_{1}, \Upsilon_{1}\right)$ for every $\Upsilon_{1} \neq \Upsilon_{2}$. Thus, $\operatorname{ESM}_{d}\left(\Upsilon_{1}, \Upsilon_{2}\right)<\operatorname{ESM}_{d}\left(\Upsilon_{1}, \Upsilon_{1}\right)$ for every $\Upsilon_{1} \neq$ $\Upsilon_{2}$.
(3) Symmetry: $d\left(\Upsilon_{1}, \Upsilon_{2}\right)=d\left(\Upsilon_{2}, \Upsilon_{1}\right)$ for every $\Upsilon_{1}$ and $\Upsilon_{2}$. Thus, $\operatorname{ESM}_{d}\left(\Upsilon_{1}, \Upsilon_{2}\right)=\operatorname{ESM}_{d}\left(\Upsilon_{2}, \Upsilon_{1}\right)$ for every $\Upsilon_{1}$ and $\Upsilon_{2}$.

(4) Triangle inequality: $d\left(\Upsilon_{1}, \Upsilon_{2}\right)+d\left(\Upsilon_{2}, \Upsilon_{3}\right) \geq d\left(\Upsilon_{1}\right.$, $\Upsilon_{3}$ ) for all $\Upsilon_{1}, \Upsilon_{2}$ and $\Upsilon_{3}$. Thus, we can say that the dissimilarities of any three $q$-RLDFSs satisfy the above properties. We can also say that if $\Upsilon_{1}$ is similar to $\Upsilon_{2}$ and $\Upsilon_{2}$ is similar to $\Upsilon_{3}$, then $\Upsilon_{1}$ should be similar to $\Upsilon_{3}$.

Now, in equation (20), we use the normalized Hamming distance (NHD) of two $q$-RLDFSs to get a new kind of SM based on NHD.

(1) $0 \leq \operatorname{ESM}_{\mathrm{NHD}}\left(\Upsilon_{1}, \Upsilon_{2}\right) \leq 1$

(2) $\operatorname{ESM}_{\mathrm{NHD}}\left(\Upsilon_{1}, \Upsilon_{2}\right)=\operatorname{ESM}_{\mathrm{NHD}}\left(\Upsilon_{1}, \Upsilon_{2}\right)$

(3) $\operatorname{ESM}_{\mathrm{NHD}}\left(\Upsilon_{1}, \Upsilon_{2}\right)=1$ iff $\Upsilon_{1}=\Upsilon_{2}$

(1) $0 \leq \operatorname{ESM}_{\mathrm{NED}}\left(\Upsilon_{1}, \Upsilon_{2}\right) \leq 1$

(2) $\operatorname{ESM}_{\mathrm{NED}}\left(\Upsilon_{1}, \Upsilon_{2}\right)=\operatorname{ESM}_{\mathrm{NED}}\left(\Upsilon_{1}, \Upsilon_{2}\right)$

(3) $\operatorname{ESM}_{\mathrm{NED}}\left(\Upsilon_{1}, \Upsilon_{2}\right)=1$ iff $\Upsilon_{1}=\Upsilon_{2}$

Definition 11. Consider two nonempty $q$-RLDFNs $\Upsilon_{1}=$ $\left(\left\langle u_{\Upsilon_{1}}\left(x_{i}\right), v_{\Upsilon_{1}}\left(x_{i}\right)\right\rangle,\left\langle a_{i}, b_{i}\right\rangle\right)$ and $\Upsilon_{2}=\left(\left\langle u_{\Upsilon_{2}}\left(x_{i}\right), v_{\Upsilon_{2}}\left(x_{i}\right)\right\rangle\right.$, $\left.\left\langle c_{i}, d_{i}\right\rangle\right)$ on the discrete nonempty set $\wp=\left(\rho_{1}, \rho_{2}, \ldots, \rho_{n}\right)$. Then, ESM based on NHD is generated by using equation (20) and given as 


$$
\operatorname{ESM}_{\mathrm{NHD}}\left(\Upsilon_{1}, \Upsilon_{2}\right)=e_{i=1}^{-(1 / 4 n)} \sum_{n}\left\{\begin{array}{c}
\left|u_{\Upsilon_{1}}^{q}\left(x_{i}\right)-u_{\Upsilon_{2}}^{q}\left(x_{i}\right)\right| \\
+\left|v_{\Upsilon_{1}}^{q}\left(x_{i}\right)-v_{\Upsilon_{2}}^{q}\left(x_{i}\right)\right|+\left|a_{i}^{q}-c_{i}^{q}\right|+\left|b_{i}^{q}-d_{i}^{q}\right|
\end{array}\right\} .
$$

Theorem 2. Consider two nonempty $q$-RLDFNs $\Upsilon_{1}=$ $\left(\left\langle u_{\Upsilon_{1}}\left(x_{i}\right), v_{\Upsilon_{1}}\left(x_{i}\right)\right\rangle,\left\langle a_{i}, b_{i}\right\rangle\right)$ and $\Upsilon_{2}=\left(\left\langle u_{\Upsilon_{2}}\left(x_{i}\right), v_{\Upsilon_{2}}\left(x_{i}\right)\right\rangle\right.$, $\left.\left\langle c_{i}, d_{i}\right\rangle\right)$ on the discrete nonempty set $\wp=\left(\rho_{1}, \rho_{2}, \ldots, \rho_{n}\right)$. Then, $\operatorname{ESM}_{N H D}\left(\Upsilon_{1}, \Upsilon_{2}\right)$ defined in equation (21) satisfies the following conditions:

Proof.

(1) We know from the definition of $q$-RLDFS that $0 \leq$ $u_{\Upsilon_{1}}\left(x_{i}\right)+v_{\Upsilon_{1}}\left(x_{i}\right)+a_{i}+b_{i} \leq 1$ and $0 \leq u_{\Upsilon_{2}}\left(x_{i}\right)+$ $v_{\Upsilon_{2}}\left(x_{i}\right)+a_{i}+b_{i} \leq 1$. Thus, we can write as

$$
\frac{1}{4 n} \sum_{i=1}^{n}\left\{\begin{array}{c}
\left|u_{\Upsilon_{1}}^{q}\left(x_{i}\right)-u_{\Upsilon_{2}}^{q}\left(x_{i}\right)\right| \\
+\left|v_{\Upsilon_{1}}^{q}\left(x_{i}\right)-v_{\Upsilon_{2}}^{q}\left(x_{i}\right)\right|+\left|a_{i}^{q}-c_{i}^{q}\right|+\left|b_{i}^{q}-d_{i}^{q}\right|
\end{array}\right\} \geq 0,
$$

and

$$
-\frac{1}{4 n} \sum_{i=1}^{n}\left\{\left|u_{\Upsilon_{1}}^{q}\left(x_{i}\right)-u_{\Upsilon_{2}}^{q}\left(x_{i}\right)\right|+\left|v_{\Upsilon_{1}}^{q}\left(x_{i}\right)-v_{\Upsilon_{2}}^{q}\left(x_{i}\right)\right|+\left|a_{i}^{q}-c_{i}^{q}\right|+\left|b_{i}^{q}-d_{i}^{q}\right|\right\} \leq 0 .
$$

We know about the exponential properties that the zero power of $e$ is equal to 1 , and the value of the

negative power of $e$ is less than 1 . In this case, we have

$$
0 \leq e^{-(1 / 4 n)} \sum_{i=1}^{n}\left\{\left|u_{\Upsilon_{1}}^{q}\left(x_{i}\right)-u_{\Upsilon_{2}}^{q}\left(x_{i}\right)\right|+\left|v_{\Upsilon_{1}}^{q}\left(x_{i}\right)-v_{\Upsilon_{2}}^{q}\left(x_{i}\right)\right|+\left|a_{i}^{q}-c_{i}^{q}\right|+\left|b_{i}^{q}-d_{i}^{q}\right|\right\} \leq 1 .
$$

Thus, we obtained that $0 \leq \operatorname{ESM}_{\mathrm{NHD}}\left(\Upsilon_{1}, \Upsilon_{2}\right) \leq 1$.

(2) The proof of this property is trivial.
(3) Suppose that $\Upsilon_{1}=\Upsilon_{2}$; then, $u_{\Upsilon_{1}}^{q}\left(x_{i}\right)=u_{\Upsilon_{2}}^{q}\left(x_{i}\right)$, $v_{\Upsilon_{1}}^{q}\left(x_{i}\right)=v_{\Upsilon_{2}}^{q}\left(x_{i}\right), a_{i}^{q}=c_{i}^{q}, b_{i}^{q}=d_{i}^{q}$. Thus, equation (14) becomes

$$
\begin{aligned}
\operatorname{ESM}_{\mathrm{NHD}}\left(\Upsilon_{1}, \Upsilon_{2}\right) \\
\\
=e^{-(1 / 4 n)} \sum_{i=1}^{n}\left\{\left|u_{\Upsilon_{1}}^{q}\left(x_{i}\right)-u_{\Upsilon_{2}}^{q}\left(x_{i}\right)\right|+\left|v_{\Upsilon_{1}}^{q}\left(x_{i}\right)-v_{\Upsilon_{2}}^{q}\left(x_{i}\right)\right|+\left|a_{i}^{q}-c_{i}^{q}\right|+\left|b_{i}^{q}-d_{i}^{q}\right|\right\} \\
=e^{0} \\
=1 .
\end{aligned}
$$

Hence, $E S M_{N H D}\left(\Upsilon_{1}, \Upsilon_{2}\right)=1$.

Next, using the normalized Euclidean distance (NED) of two $q$-RLDFSs in equation (21), we will get the new type of SM based on ED.
Definition 12. Consider two nonempty $q$-RLDFNs $\Upsilon_{1}=$ $\left(\left\langle u_{\Upsilon_{1}}\left(x_{i}\right), v_{\Upsilon_{1}}\left(x_{i}\right)\right\rangle,\left\langle a_{i}, b_{i}\right\rangle\right)$ and $\Upsilon_{2}=\left(\left\langle u_{\Upsilon_{2}}\left(x_{i}\right), v_{\Upsilon_{2}}\left(x_{i}\right)\right\rangle\right.$, $\left.\left\langle c_{i}, d_{i}\right\rangle\right)$ on the discrete nonempty set $\wp=\left(\rho_{1}, \rho_{2}, \ldots, \rho_{n}\right)$. Then, ESM based on NED is generated. So, the ESM based on NED is given in the following equation:

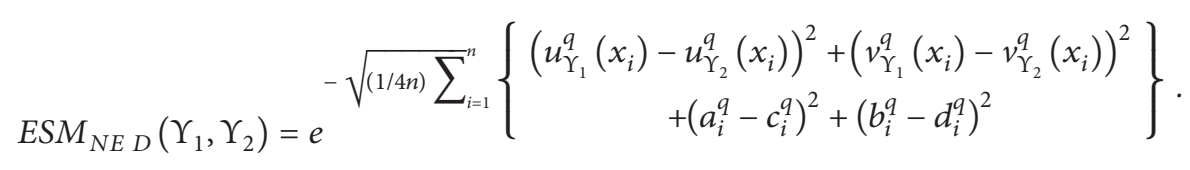


Theorem 12. Consider two nonempty $q$-RLDFNs $\Upsilon_{1}=$ $\left(\left\langle u_{\Upsilon_{1}}\left(x_{i}\right), v_{\Upsilon_{1}}\left(x_{i}\right)\right\rangle,\left\langle a_{i}, b_{i}\right\rangle\right)$ and $\Upsilon_{2}=\left(\left\langle u_{\Upsilon_{2}}\left(x_{i}\right), v_{\Upsilon_{2}}\left(x_{i}\right)\right\rangle\right.$, $\left.\left\langle c_{i}, d_{i}\right\rangle\right)$ on the discrete nonempty set $\wp=\left(\rho_{1}, \rho_{2}, \ldots, \rho_{n}\right)$. Then, $\operatorname{ESM}_{N E D}\left(\Upsilon_{1}, \Upsilon_{2}\right)$ defined in equation (26) satisfies the following conditions:
Proof

(1) Given that $\Upsilon_{1}$ and $\Upsilon_{2}$ are the two $q$-RLDFNs and as $0 \leq u_{\Upsilon_{1}}\left(x_{i}\right)+v_{\Upsilon_{1}}\left(x_{i}\right)+a_{i}+b_{i} \leq 1$ and $0 \leq u_{\Upsilon_{2}}\left(x_{i}\right)$ $+v_{\Upsilon_{2}}\left(x_{i}\right)+a_{i}+b_{i} \leq 1$, it implies that

$$
e^{\sqrt{(1 / 4 n) \sum_{i=1}^{n}\left\{\left(u_{\Upsilon_{1}}^{q}\left(x_{i}\right)-u_{\Upsilon_{2}}^{q}\left(x_{i}\right)\right)^{2}+\left(v_{\Upsilon_{1}}^{q}\left(x_{i}\right)-v_{\Upsilon_{2}}^{q}\left(x_{i}\right)\right)^{2}+\left(a_{i}^{q}-c_{i}^{q}\right)^{2}+\left(b_{i}^{q}-d_{i}^{q}\right)^{2}\right\}}} \geq 0,
$$

and

$$
\left.e \sqrt{(1 / 4 n) \sum_{i=1}^{n}\left\{\begin{array}{c}
\left(u_{\Upsilon_{1}}^{q}\left(x_{i}\right)-u_{\Upsilon_{2}}^{q}\left(x_{i}\right)\right)^{2}+\left(v_{\Upsilon_{1}}^{q}\left(x_{i}\right)-v_{\Upsilon_{2}}^{q}\left(x_{i}\right)\right)^{2} \\
+\left(a_{i}^{q}-c_{i}^{q}\right)^{2}+\left(b_{i}^{q}-d_{i}^{q}\right)^{2}
\end{array}\right.}\right\}_{\leq 0 .}
$$

We also know about the exponential properties that the zero power of $e$ is equal to 1 , and the value of the negative power of $e$ is less than 1 . Then, we have

$$
\begin{aligned}
\operatorname{ESM}_{\mathrm{NED}} & \left(\Upsilon_{1}, \Upsilon_{2}\right) \\
& \\
& -\sqrt{(1 / 4 n) \sum_{i=1}^{n}\left\{\begin{array}{c}
\left(u_{\Upsilon_{1}}^{q}\left(x_{i}\right)-u_{\Upsilon_{2}}^{q}\left(x_{i}\right)\right)^{2}+\left(v_{\Upsilon_{1}}^{q}\left(x_{i}\right)-v_{\Upsilon_{2}}^{q}\left(x_{i}\right)\right)^{2} \\
+\left(a_{i}^{q}-c_{i}^{q}\right)^{2}+\left(b_{i}^{q}-d_{i}^{q}\right)^{2}
\end{array}\right\}} \\
& =e \\
& =e^{0} \\
& =1 .
\end{aligned}
$$

Hence, $\operatorname{ESM}_{\mathrm{ED}}\left(\Upsilon_{1}, \Upsilon_{2}\right)=1$.

(2) The proof of this property is followed by using the symmetric property of NED. So, we omit the proof here.
(3) Suppose that $\Upsilon_{1}=\Upsilon_{2}$; then, $u_{\Upsilon_{1}}^{q}\left(x_{i}\right)=u_{\Upsilon_{2}}^{q}\left(x_{i}\right)$, $v_{\Upsilon_{1}}^{q}\left(x_{i}\right)=v_{\Upsilon_{2}}^{q}\left(x_{i}\right), a_{i}^{q}=c_{i}^{q}, b_{i}^{q}=d_{i}^{q}$. Thus, equation (26) becomes

$$
\begin{aligned}
\operatorname{ESM}_{\mathrm{NED}} & \left(\Upsilon_{1}, \Upsilon_{2}\right) \\
& =-\sqrt{(1 / 4 n) \sum_{i=1}^{n}\left\{\begin{array}{c}
\left.\left(u_{\Upsilon_{1}}^{q}\left(x_{i}\right)-u_{\Upsilon_{2}}^{q}\left(x_{i}\right)\right)^{2}+\left(v_{\Upsilon_{1}}^{q}\left(x_{i}\right)-v_{\Upsilon_{2}}^{q}\left(x_{i}\right)\right)^{2}\right\} \\
+\left(a_{i}^{q}-c_{i}^{q}\right)^{2}+\left(b_{i}^{q}-d_{i}^{q}\right)^{2}
\end{array}\right\}} \\
& =e^{-} \\
& =e^{0} \\
& =1 .
\end{aligned}
$$

Hence, $\operatorname{ESM}_{\mathrm{NED}}\left(\Upsilon_{1}, \Upsilon_{2}\right)=1$.

Next, we use the weight of each $x_{i}$ with the weight vector $w$ $=\left(w_{1}, w_{2}, \ldots, w_{n}\right)^{T}$ subject to $w_{i} \in[0,1]$ such that $\sum_{i=1}^{n} w_{i}=1$.
Then, we propose a new ESM, called the weighted exponential similarity measure (WESM) of $\Upsilon_{1}$ and $\Upsilon_{2}$ which is denoted by $\mathrm{WESM}_{\mathrm{NHD}}\left(\Upsilon_{1}, \Upsilon_{2}\right)$ and $\mathrm{WESM}_{\mathrm{NED}}\left(\Upsilon_{1}, \Upsilon_{2}\right)$, respectively. 
Definition 13. Consider two nonempty $q$-RLDFNs $\Upsilon_{1}=$ $\left(\left\langle u_{\Upsilon_{1}}\left(x_{i}\right), v_{\Upsilon_{1}}\left(x_{i}\right)\right\rangle,\left\langle a_{i}, b_{i}\right\rangle\right)$ and $\Upsilon_{2}=\left(\left\langle u_{\Upsilon_{2}}\left(x_{i}\right), v_{\Upsilon_{2}}\left(x_{i}\right)\right\rangle\right.$, $\left.\left\langle c_{i}, d_{i}\right\rangle\right)$ on the discrete nonempty set $\wp=\left(\rho_{1}, \rho_{2}, \ldots, \rho_{n}\right)$.
Then, ESM based on NHD and NED is denoted by $\mathrm{WESM}_{\mathrm{NHD}}\left(\Upsilon_{1}, \Upsilon_{2}\right)$ and $\operatorname{WESM}_{\mathrm{NED}}\left(\Upsilon_{1}, \Upsilon_{2}\right)$, given in equations (31) and (32), respectively.

$$
\left.\mathrm{WESM}_{\mathrm{NHD}}\left(\Upsilon_{1}, \Upsilon_{2}\right)=e^{-\left\{(1 / 4 n) \sum_{i=1}^{n} w_{i}\right.}\left(\left[\begin{array}{c}
\left|u_{\Upsilon_{1}}^{q}\left(x_{i}\right)-u_{\Upsilon_{2}}^{q}\left(x_{i}\right)\right|+\left|v_{\Upsilon_{1}}^{q}\left(x_{i}\right)-v_{\Upsilon_{2}}^{q}\left(x_{i}\right)\right| \\
+\left|a_{i}^{q}-c_{i}^{q}\right|+\left|b_{i}^{q}-d_{i}^{q}\right|
\end{array}\right]\right)\right\} .
$$

The weighted ESM based on NED is given as

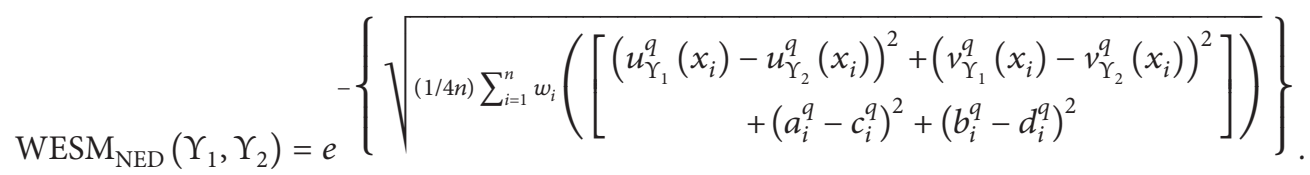

\section{Similarity Measure of $q$-RLDFS Based on Cosine and Cotangent Functions}

This section is used for the SM based on cosine and cotangent functions for $q$-RLDFSs. The proposed SM is also known as a cosine similarity measure (CSM) and cotangent similarity measure (cotSM) between two $q$-RLDFSs $\Upsilon_{1}$ and $\Upsilon_{2}$. The CSM is generated by using the cosine function, and cotSM is generated by using the cotangent function. This section has two sections: one is for CSM and another is for cotSM.
5.1. Cosine Similarity Measure of $q-R L D F S s$. In this section, the authors use the cosine function to develop a CSM for two $q$-RLDFSs $\Upsilon_{1}$ and $\Upsilon_{2}$. We also develop the weighted CSM for two $q$-RLDFSs $\Upsilon_{1}$ and $\Upsilon_{2}$ and also prove some properties of the CSM and WCSM with the distance measure.

Definition 14. Consider two nonempty $q$-RLDFNs $\Upsilon_{1}=$ $\left(\left\langle u_{\Upsilon_{1}}\left(x_{i}\right), v_{\Upsilon_{1}}\left(x_{i}\right)\right\rangle,\left\langle a_{i}, b_{i}\right\rangle\right)$ and $\Upsilon_{2}=\left(\left\langle u_{\Upsilon_{2}}\left(x_{i}\right), v_{\Upsilon_{2}}\left(x_{i}\right)\right\rangle\right.$, $\left.\left\langle c_{i}, d_{i}\right\rangle\right)$ on the discrete nonempty set $\wp=\left(\rho_{1}, \rho_{2}, \ldots, \rho_{n}\right)$. Then, the cosine similarity measure is denoted by CSM $\left(\Upsilon_{1}, \Upsilon_{2}\right)$ and given as

$$
\begin{aligned}
\operatorname{CSM}\left(\Upsilon_{1}, \Upsilon_{2}\right)=\frac{1}{n} \sum_{i=1}^{n} & \frac{u_{\Upsilon_{1}}^{q}(x) u_{\Upsilon_{2}}^{q}\left(x_{i}\right)+v_{\Upsilon_{1}}\left(x_{i}\right) v_{\Upsilon_{2}}\left(x_{i}\right)}{} \\
& +a_{i}^{q} c_{i}^{q}+b_{i}^{q} d_{i}^{q} \\
& \sqrt{\left(u_{\Upsilon_{1}}^{q}\left(x_{i}\right)\right)^{2}+\left(v_{\Upsilon_{1}}^{q}\left(x_{i}\right)\right)^{2}+\left(a_{i}^{q}\right)^{2}+\left(c_{i}^{q}\right)^{2}} \\
& \sqrt{\left(u_{\Upsilon_{2}}^{q}\left(x_{i}\right)\right)^{2}+\left(v_{\Upsilon_{2}}^{q}\left(x_{i}\right)\right)^{2}+\left(b_{i}^{q}\right)^{2}+\left(d_{i}^{q}\right)^{2}}
\end{aligned}
$$

The CSM of three $q$-RLDFSs $\Upsilon_{1}, \Upsilon_{2}$, and $\Upsilon_{3}$ satisfied the following properties:

(1) $0 \leq \operatorname{CSM}\left(\Upsilon_{1}, \Upsilon_{2}\right) \leq 1$.

(2) $\operatorname{CSM}\left(\Upsilon_{1}, \Upsilon_{2}\right) \leq \operatorname{CSM}\left(\Upsilon_{2}, \Upsilon_{1}\right)$.

(3) $\operatorname{CSM}\left(\Upsilon_{1}, \Upsilon_{2}\right)=1 \Leftrightarrow \Upsilon_{1}=\Upsilon_{2}$.

(4) Let $\Upsilon_{1} \subseteq \Upsilon_{2} \subseteq \Upsilon_{3}$. Then, $\operatorname{CSM}\left(\Upsilon_{1}, \Upsilon_{3}\right) \leq \operatorname{CSM}\left(\Upsilon_{1}, \Upsilon_{2}\right)$ and $\operatorname{CSM}\left(\Upsilon_{1}, \Upsilon_{3}\right) \leq \operatorname{CSM}\left(\Upsilon_{2}, \Upsilon_{3}\right)$.

Proof. The first two properties are obvious, so we omit the proof here and prove the last two properties.

(3): assume that $\Upsilon_{1}=\Upsilon_{2}$; then, $u_{\Upsilon_{1}}^{q}(x)=u_{\Upsilon_{2}}^{q}\left(x_{i}\right), v_{\Upsilon_{1}}^{q}(x)$ $=v_{\Upsilon_{2}}^{q}\left(x_{i}\right), a^{q}=b^{q}$ and $c^{q}=d^{q}$ for all $1 \leq i \leq n$. We have

$$
\begin{aligned}
& \operatorname{CSM}\left(\Upsilon_{1}, \Upsilon_{1}\right)= \frac{1}{n} \sum_{i=1}^{n} \frac{u_{\Upsilon_{1}}^{q}\left(x_{i}\right) u_{\Upsilon_{1}}^{q}(x)+v_{\Upsilon_{1}}^{q}\left(x_{i}\right) v_{\Upsilon_{1}}^{q}\left(x_{i}\right)+a_{i}^{q} a_{i}^{q}+b_{i}^{q} b_{i}^{q}}{\sqrt{\left(u_{\Upsilon_{1}}^{q}\left(x_{i}\right)\right)^{2}+\left(v_{\Upsilon_{1}}^{q}\left(x_{i}\right)\right)^{2}+\left(a_{i}^{q}\right)^{2}+\left(a_{i}^{q}\right)^{2}}} \\
& \sqrt{\left(u_{\Upsilon_{2}}^{q}\left(x_{i}\right)\right)^{2}+\left(v_{\Upsilon_{2}}^{q}\left(x_{i}\right)\right)^{2}+\left(b_{i}^{q}\right)^{2}+\left(b_{i}^{q}\right)^{2}} \\
&=\frac{1}{n} \sum_{i=1}^{n} \frac{\left(u_{\Upsilon_{1}}^{q}\left(x_{i}\right)\right)^{2}+\left(v_{\Upsilon_{1}}^{q}\left(x_{i}\right)\right)^{2}+\left(a_{i}^{q}\right)^{2}+\left(b_{i}^{q}\right)^{2}}{\left(u_{\Upsilon_{1}}^{q}\left(x_{i}\right)\right)^{2}+\left(v_{\Upsilon_{1}}^{q}\left(x_{i}\right)\right)^{2}+\left(a_{i}^{q}\right)^{2}+\left(c_{i}^{q}\right)^{2}} \\
&=1 .
\end{aligned}
$$

(4): now, to prove the last property, let $\Upsilon_{1} \subseteq \Upsilon_{2} \subseteq \Upsilon_{3}$, so the angle of $\Upsilon_{1}, \Upsilon_{3}$ is larger than the angle of $\Upsilon_{1}, \Upsilon_{2}$ and 
$\Upsilon_{1}, \Upsilon_{3}$. Thus, from the fact, we can write as $\operatorname{CSM}\left(\Upsilon_{1}, \Upsilon_{3}\right) \leq$ $\operatorname{CSM}\left(\Upsilon_{1}, \Upsilon_{2}\right)$ and $\operatorname{CSM}\left(\Upsilon_{1}, \Upsilon_{3}\right) \leq \operatorname{CSM}\left(\Upsilon_{2}, \Upsilon_{3}\right)$.

In the following, we present a relation between the distance measure (DsM) and CSM. We explore the DsM between two $q$-RLDFSs using the CSM.

Definition 15. Consider two nonempty $q$-RLDFNs $\Upsilon_{1}=$ $\left(\left\langle u_{\Upsilon_{1}}\left(x_{i}\right), v_{\Upsilon_{1}}\left(x_{i}\right)\right\rangle,\left\langle a_{i}, b_{i}\right\rangle\right)$ and $\Upsilon_{2}=\left(\left\langle u_{\Upsilon_{2}}\left(x_{i}\right), v_{\Upsilon_{2}}\left(x_{i}\right)\right\rangle\right.$, $\left.\left\langle c_{i}, d_{i}\right\rangle\right)$ on the discrete nonempty $\wp=\left(\rho_{1}, \rho_{2}, \ldots, \rho_{n}\right)$. Then, the DsM between two $q$-RLDFSs is given by the following equation:

$$
d\left(\Upsilon_{1}, \Upsilon_{2}\right)=\cos ^{-1}\left(\operatorname{CSM}\left(\Upsilon_{1}, \Upsilon_{2}\right)\right)
$$

Now, we will prove that equation (35) satisfies the conditions of the DsM:

(1) $0 \leq \operatorname{CSM}\left(\Upsilon_{1}, \Upsilon_{2}\right) \leq 1$; then, $d\left(\Upsilon_{1}, \Upsilon_{2}\right) \geq 0$
(2) $\operatorname{CSM}\left(\Upsilon_{1}, \Upsilon_{2}\right)=\operatorname{CSM}\left(\Upsilon_{2}, \Upsilon_{1}\right)$; then, $d\left(\Upsilon_{1}, \Upsilon_{2}\right)=d$ $\left(\Upsilon_{2}, \Upsilon_{1}\right)$

(3) $\operatorname{CSM}\left(\Upsilon_{1}, \Upsilon_{2}\right)=1$; if $\Upsilon_{1}=\Upsilon_{2}$, then $d\left(\Upsilon_{1}, \Upsilon_{2}\right)=0$

(4) If $\Upsilon_{1} \subseteq \Upsilon_{2} \subseteq \Upsilon_{3}$, then $d\left(\Upsilon_{1}, \Upsilon_{3}\right) \leq d\left(\Upsilon_{1}, \Upsilon_{2}\right)+\left(\Upsilon_{2}, \Upsilon_{3}\right)$

Proof. The first three properties of DsM are obvious; we only prove the last property of equation (35); consider $\Upsilon_{1}, \Upsilon_{2}$, and $\Upsilon_{3}$ such that $\Upsilon_{1} \subseteq \Upsilon_{2} \subseteq \Upsilon_{3}$. Then, DsM of $\Upsilon_{1}, \Upsilon_{2}$, and $\Upsilon_{3}$ is given as

$$
\begin{aligned}
& d\left(\Upsilon_{1}, \Upsilon_{2}\right)=\cos ^{-1}\left(\operatorname{CSM}\left(\Upsilon_{1}, \Upsilon_{2}\right)\right), \\
& d\left(\Upsilon_{2}, \Upsilon_{3}\right)=\cos ^{-1}\left(\operatorname{CSM}\left(\Upsilon_{2}, \Upsilon_{3}\right)\right), \\
& d\left(\Upsilon_{1}, \Upsilon_{3}\right)=\cos ^{-1}\left(\operatorname{CSM}\left(\Upsilon_{1}, \Upsilon_{3}\right)\right) .
\end{aligned}
$$

Therefore, we can write the following equations from the above equations as

$$
\begin{aligned}
& \operatorname{CSM}\left(\Upsilon_{1}, \Upsilon_{2}\right)=\frac{1}{n} \sum_{i=1}^{n} \frac{u_{\Upsilon_{1}}^{q}(x) u_{\Upsilon_{2}}^{q}\left(x_{i}\right)+v_{\Upsilon_{1}}^{q}\left(x_{i}\right) v_{\Upsilon_{2}}^{q}\left(x_{i}\right)+a_{i}^{q} c_{i}^{q}+b_{i}^{q} d_{i}^{q}}{\sqrt{\left(u_{\Upsilon_{1}}^{q}\left(x_{i}\right)\right)^{2}+\left(v_{\Upsilon_{1}}^{q}\left(x_{i}\right)\right)^{2}+\left(a_{i}^{q}\right)^{2}+\left(c_{i}^{q}\right)^{2}}}, \\
& \sqrt{\left(u_{\Upsilon_{2}}^{q}\left(x_{i}\right)\right)^{2}+\left(v_{\Upsilon_{2}}^{q}\left(x_{i}\right)\right)^{2}+\left(b_{i}^{q}\right)^{2}+\left(d_{i}^{q}\right)^{2}} \\
& \operatorname{CSM}\left(\Upsilon_{2}, \Upsilon_{3}\right)=\frac{1}{n} \sum_{i=1}^{n} \frac{u_{\Upsilon_{2}}^{q}(x) u_{\Upsilon_{3}}^{q}\left(x_{i}\right)+v_{\Upsilon_{2}}\left(x_{i}\right) v_{\Upsilon_{3}}\left(x_{i}\right)+c_{i}^{q} e_{i}^{q}+d_{i}^{q} f_{i}^{q}}{\sqrt{\left(u_{\Upsilon_{2}}^{q}\left(x_{i}\right)\right)^{2}+\left(v_{\Upsilon_{3}}^{q}\left(x_{i}\right)\right)^{2}+\left(c_{i}^{q}\right)^{2}+\left(e_{i}^{q}\right)^{2}}}, \\
& \sqrt{\left(u_{\Upsilon_{2}}^{q}\left(x_{i}\right)\right)^{2}+\left(v_{\Upsilon_{2}}^{q}\left(x_{i}\right)\right)^{2}+\left(d_{i}^{q}\right)^{2}+\left(f_{i}^{q}\right)^{2}} \\
& \operatorname{CSM}\left(\Upsilon_{1}, \Upsilon_{3}\right)=\frac{1}{n} \sum_{i=1}^{n} \frac{u_{\Upsilon_{1}}^{q}(x) u_{\Upsilon_{3}}^{q}\left(x_{i}\right)+v_{\Upsilon_{1}}\left(x_{i}\right) v_{\Upsilon_{3}}\left(x_{i}\right)+a_{i}^{q} e_{i}^{q}+b_{i}^{q} f_{i}^{q}}{\sqrt{\left(u_{\Upsilon_{1}}^{q}\left(x_{i}\right)\right)^{2}+\left(v_{\Upsilon_{3}}^{q}\left(x_{i}\right)\right)^{2}+\left(a_{i}^{q}\right)^{2}+\left(e_{i}^{q}\right)^{2}}} . \\
& \sqrt{\left(u_{\Upsilon_{1}}^{q}\left(x_{i}\right)\right)^{2}+\left(v_{\Upsilon_{2}}^{q}\left(x_{i}\right)\right)^{2}+\left(b_{i}^{q}\right)^{2}+\left(f_{i}^{q}\right)^{2}}
\end{aligned}
$$

Now, consider weight $w_{i}$ for each $x_{i}$ with the weight vector for all values of $x_{i}$. Then, we propose the weighted CSM for two $q$-RLDFSs.
Definition 16. Consider two nonempty $q$-RLDFNs $\Upsilon_{1}=$ $\left(\left\langle u_{\Upsilon_{1}}\left(x_{i}\right), v_{\Upsilon_{1}}\left(x_{i}\right)\right\rangle,\left\langle a_{i}, b_{i}\right\rangle\right)$ and $\Upsilon_{2}=\left(\left\langle u_{\Upsilon_{2}}\left(x_{i}\right), v_{\Upsilon_{2}}\left(x_{i}\right)\right\rangle\right.$, $\left.\left\langle c_{i}, d_{i}\right\rangle\right)$ on the discrete nonempty set $\wp=\left(\rho_{1}, \rho_{2}, \ldots, \rho_{n}\right)$. Then, the weighted cosine similarity measure is denoted by $\operatorname{WCSM}\left(\Upsilon_{1}, \Upsilon_{2}\right)$ and given as

$$
\begin{gathered}
\operatorname{WCSM}\left(\Upsilon_{1}, \Upsilon_{2}\right)=\sum_{i=1}^{n} w_{i} \frac{u_{\Upsilon_{1}}^{q}(x) u_{\Upsilon_{2}}^{q}\left(x_{i}\right)+v_{\Upsilon_{1}}\left(x_{i}\right) v_{\Upsilon_{2}}\left(x_{i}\right)+a_{i}^{q} c_{i}^{q}+b_{i}^{q} d_{i}^{q}}{\sqrt{\left(u_{\Upsilon_{1}}^{q}\left(x_{i}\right)\right)^{2}+\left(v_{\Upsilon_{1}}^{q}\left(x_{i}\right)\right)^{2}+\left(a_{i}^{q}\right)^{2}+\left(c_{i}^{q}\right)^{2}}}, \\
\sqrt{\left(u_{\Upsilon_{2}}^{q}\left(x_{i}\right)\right)^{2}+\left(v_{\Upsilon_{2}}^{q}\left(x_{i}\right)\right)^{2}+\left(b_{i}^{q}\right)^{2}+\left(d_{i}^{q}\right)^{2}}
\end{gathered}
$$

where $w=\left(w_{1}, w_{2}, \ldots, w_{n}\right)^{T}$ is a weight vector of each $x_{i}$ such that $w_{i} \in[0,1]$ and $\sum_{i=1}^{n} w_{i}=1$. If we take $w_{i}=1 / n$, then equation (40) becomes equation (33). The WCSM is a generalized form of the CSM. The WCSM is also satisfied for two $q$-RLDFSs.
The CSM of three $q$-RLDFNs $\Upsilon_{1}, \Upsilon_{2}$, and $\Upsilon_{3}$ satisfied the following properties:

(1) $0 \leq \operatorname{WCSM}\left(\Upsilon_{1}, \Upsilon_{2}\right) \leq 1$

(2) $\operatorname{WCSM}\left(\Upsilon_{1}, \Upsilon_{2}\right) \leq \operatorname{WCSM}\left(\Upsilon_{2}, \Upsilon_{1}\right)$ 
(3) $\operatorname{WCSM}\left(\Upsilon_{1}, \Upsilon_{2}\right)=1 \Leftrightarrow \Upsilon_{1}=\Upsilon_{2}$

Proof. The proof follows from the proof of the properties of equation (35).

Now, we will use the cosine function to propose new SMs and WSMs based on the cosine function. The similarity measure based on the cosine function is given in equations (41) and (42).

Definition 17. The SMs based on the cosine function for two $q$-RLDFNs $\Upsilon_{1}$ and $\Upsilon_{2}$ are denoted by $\operatorname{SM}_{\text {cos }}\left(\Upsilon_{1}, \Upsilon_{2}\right)$ and defined by the following equation:

$\mathrm{SM}_{\cos ^{1}}\left(\Upsilon_{1}, \Upsilon_{2}\right)=\frac{1}{n} \sum_{i=1}^{n} \cos \left[\frac{\pi}{2}\left(\begin{array}{c}\left|u_{\Upsilon_{1}}^{q}\left(x_{i}\right)-u_{\Upsilon_{2}}^{q}\left(x_{i}\right)\right| \\ \vee\left|v_{\Upsilon_{1}}^{q}\left(x_{i}\right)-v_{\Upsilon_{2}}^{q}\left(x_{i}\right)\right| \\ \vee\left|a_{i}^{q}-c_{i}^{q}\right| \vee\left|b_{i}^{q}-d_{i}^{q}\right|\end{array}\right)\right]$,

$\operatorname{SM}_{\cos ^{2}}\left(\Upsilon_{1}, \Upsilon_{2}\right)=\frac{1}{n} \sum_{i=1}^{n} \cos \left[\frac{\pi}{4}\left(\begin{array}{c}\left|u_{\Upsilon_{1}}^{q}\left(x_{i}\right)-u_{\Upsilon_{2}}^{q}\left(x_{i}\right)\right| \\ +\left|v_{\Upsilon_{1}}^{q}\left(x_{i}\right)-v_{\Upsilon_{2}}^{q}\left(x_{i}\right)\right| \\ +\left|a_{i}^{q}-c_{i}^{q}\right|+\left|b_{i}^{q}-d_{i}^{q}\right|\end{array}\right)\right]$.

Also, we prove the following properties for equations (41) and (42):

(1) $0 \leq \operatorname{SM}_{\cos ^{1,2}}\left(\Upsilon_{1}, \Upsilon_{2}\right) \leq 1$

(2) $\operatorname{SM}_{\cos ^{1,2}}\left(\Upsilon_{1}, \Upsilon_{2}\right)=\operatorname{SM}_{\cos ^{1,2}}\left(\Upsilon_{2}, \Upsilon_{1}\right)$

(3) $\operatorname{SM}_{\cos ^{1,2}}\left(\Upsilon_{1}, \Upsilon_{2}\right)=1$ iff $\Upsilon_{1}=\Upsilon_{2}$

(4) If $\Upsilon_{1} \subseteq \Upsilon_{2} \subseteq \Upsilon_{3}$, then $\operatorname{SM}_{\cos ^{1,2}}\left(\Upsilon_{1}, \Upsilon_{3}\right) \leq \mathrm{SM}_{\cos ^{1,2}}$ $\left(\Upsilon_{1}, \Upsilon_{2}\right)$ and $\mathrm{SM}_{\cos ^{1,2}}\left(\Upsilon_{1}, \Upsilon_{3}\right) \leq \operatorname{SM}_{\cos ^{1,2}}\left(\Upsilon_{2}, \Upsilon_{3}\right)$

Proof.

(1) Since the cosine function values lie between the closed interval 0 and 1 and based on the cosine function, the $q$-RLDF cosine similarity measures are also within $[0,1]$. Thus, the values of $\mathrm{SM}_{\cos ^{1,2}}\left(\Upsilon_{1}, \Upsilon_{2}\right)$ must lie between 0 and 1 .

(2) This is easily proven that $\operatorname{SM}_{\cos ^{1,2}}\left(\Upsilon_{1}, \Upsilon_{2}\right)=$ $\mathrm{SM}_{\cos ^{1,2}}\left(\Upsilon_{2}, \Upsilon_{1}\right)$.

(3) This is easily proven such that $\operatorname{SM}_{\cos ^{1,2}}\left(\Upsilon_{1}, \Upsilon_{2}\right)=1$.

(4) Consider three $q$-RLDFNs, such as $\Upsilon_{1} \subseteq \Upsilon_{2} \subseteq \Upsilon_{3}$; then,

$$
\begin{aligned}
u_{\Upsilon_{1}}^{q}\left(x_{i}\right) & \leq u_{\Upsilon_{2}}^{q}\left(x_{i}\right) \leq u_{\Upsilon_{3}}^{q}\left(x_{i}\right), \\
v_{\Upsilon_{1}}^{q}\left(x_{i}\right) & \geq v_{\Upsilon_{2}}^{q}\left(x_{i}\right) \geq v_{\Upsilon_{3}}^{q}\left(x_{i}\right), \\
a_{i}^{q} & \leq c_{i}^{q} \leq e_{i}^{q} \text { an } b_{i}^{q} \geq d_{i}^{q} \geq f_{i}^{q} .
\end{aligned}
$$

Then,

$$
\begin{aligned}
\left|u_{\Upsilon_{1}}^{q}\left(x_{i}\right)-u_{\Upsilon_{2}}^{q}\left(x_{i}\right)\right| & \leq\left|u_{\Upsilon_{1}}^{q}\left(x_{i}\right)-u_{\Upsilon_{2}}^{q}\left(x_{i}\right)\right|, \\
\left|u_{\Upsilon_{2}}^{q}\left(x_{i}\right)-u_{\Upsilon_{3}}^{q}\left(x_{i}\right)\right| & \leq\left|u_{\Upsilon_{1}}^{q}\left(x_{i}\right)-u_{\Upsilon_{3}}^{q}\left(x_{i}\right)\right|, \\
\left|v_{\Upsilon_{1}}^{q}\left(x_{i}\right)-v_{\Upsilon_{2}}^{q}\left(x_{i}\right)\right| & \leq\left|v_{\Upsilon_{1}}^{q}\left(x_{i}\right)-v_{\Upsilon_{2}}^{q}\left(x_{i}\right)\right|, \\
\left|v_{\Upsilon_{2}}^{q}\left(x_{i}\right)-v_{\Upsilon_{3}}^{q}\left(x_{i}\right)\right| & \leq\left|v_{\Upsilon_{1}}^{q}\left(x_{i}\right)-v_{\Upsilon_{3}}^{q}\left(x_{i}\right)\right|, \\
\left|a_{i}^{q}-c_{i}^{q}\right| & \leq\left|a_{i}^{q}-e_{i}^{q}\right| \text { and }\left|c_{i}^{q}-e_{i}^{q}\right| \leq\left|a_{i}^{q}-e_{i}^{q}\right|, \\
\left|b_{i}^{q}-d_{i}^{q}\right| & \leq\left|b_{i}^{q}-f_{i}^{q}\right| \text { and }\left|d_{i}^{q}-f_{i}^{q}\right| \leq\left|b_{i}^{q}-f_{i}^{q}\right| .
\end{aligned}
$$

We know that the cosine function is a decreasing function in $[0, \pi / 2]$. Thus, $\operatorname{SM}_{\cos ^{1,2}}\left(\Upsilon_{1}, \Upsilon_{3}\right) \leq \operatorname{SM}_{\cos ^{1,2}}\left(\Upsilon_{1}, \Upsilon_{2}\right)$ and $\operatorname{SM}_{\cos ^{1,2}}\left(\Upsilon_{1}, \Upsilon_{3}\right) \leq \operatorname{SM}_{\cos ^{1,2}}\left(\Upsilon_{2}, \Upsilon_{3}\right)$.

Now, consider weight $w_{i}$ for each $x_{i}$ with the weight vector for all values of $x_{i}$. Then, we propose the weighted SM for two $q$-RLDFNs based on the cosine function.

Definition 18. Consider two nonempty $q$-RLDFNs $\Upsilon_{1}=$ $\left(\left\langle u_{\Upsilon_{1}}\left(x_{i}\right), v_{\Upsilon_{1}}\left(x_{i}\right)\right\rangle,\left\langle a_{i}, b_{i}\right\rangle\right)$ and $\Upsilon_{2}=\left(\left\langle u_{\Upsilon_{2}}\left(x_{i}\right), v_{\Upsilon_{2}}\left(x_{i}\right)\right\rangle\right.$, $\left.\left\langle c_{i}, d_{i}\right\rangle\right)$ on the discrete nonempty set $\wp=\left(\rho_{1}, \rho_{2}, \ldots, \rho_{n}\right)$. Then, the weighted cosine similarity measure based on the cosine function is denoted by $\mathrm{WSM}_{\cos ^{1,2}}\left(\Upsilon_{1}, \Upsilon_{2}\right)$ and given as

$\mathrm{WSM}_{\cos ^{1}}\left(\Upsilon_{1}, \Upsilon_{2}\right)=\sum_{i=1}^{n} w_{i} \cos \left[\frac{\pi}{2}\left(\begin{array}{c}\left|u_{\Upsilon_{1}}^{q}\left(x_{i}\right)-u_{\Upsilon_{2}}^{q}\left(x_{i}\right)\right| \\ \vee\left|v_{\Upsilon_{1}}^{q}\left(x_{i}\right)-v_{\Upsilon_{2}}^{q}\left(x_{i}\right)\right| \\ \vee\left|a_{i}^{q}-c_{i}^{q}\right| \vee\left|b_{i}^{q}-d_{i}^{q}\right|\end{array}\right)\right]$,

$$
\operatorname{WSM}_{\cos ^{2}}\left(\Upsilon_{1}, \Upsilon_{2}\right)=\sum_{i=1}^{n} w_{i} \cos \left[\frac{\pi}{4}\left(\begin{array}{c}
\left|u_{\Upsilon_{1}}^{q}\left(x_{i}\right)-u_{\Upsilon_{2}}^{q}\left(x_{i}\right)\right| \\
+\mid v_{\Upsilon_{1}}^{q}\left(x_{i}\right)-v_{\Upsilon_{2}}^{q}\left(x_{i}\right) \\
+\left|a_{i}^{q}-c_{i}^{q}\right|+\left|b_{i}^{q}-d_{i}^{q}\right|
\end{array}\right)\right]
$$

where $w=\left(w_{1}, w_{2}, \ldots, w_{n}\right)^{T}$ is a weight vector of each $x_{i}$ such that $w_{i} \in[0,1]$ and $\sum_{i=1}^{n} w_{i}=1$. If we take $w_{i}=1 / n$, then equations (45) and (46) become equations (41) and (42). The WCSM is a generalized form of the CSM. The WCSM is also satisfied for two $q$-RLDFNs.

The WCSM of three $q$-RLDFNs $\Upsilon_{1}, \Upsilon_{2}$, and $\Upsilon_{3}$ satisfied the following properties:

(1) $0 \leq \operatorname{WSM}_{\cos ^{1,2}}\left(\Upsilon_{1}, \Upsilon_{2}\right) \leq 1$

(2) $\operatorname{WSM}_{\cos ^{1,2}}\left(\Upsilon_{1}, \Upsilon_{2}\right)=\operatorname{WSM}_{\cos ^{1,2}}\left(\Upsilon_{2}, \Upsilon_{1}\right)$

(3) $\mathrm{WSM}_{\cos ^{1,2}}\left(\Upsilon_{1}, \Upsilon_{2}\right)=1 \Leftrightarrow \Upsilon_{1}=\Upsilon_{2}$

(4) If $\Upsilon_{1} \subseteq \Upsilon_{2} \subseteq \Upsilon_{3}$, then $\mathrm{WSM}_{\cos ^{1,2}}\left(\Upsilon_{1}, \Upsilon_{3}\right) \leq \mathrm{WSM}_{\cos ^{1,2}}$ $\left(\Upsilon_{1}, \Upsilon_{2}\right)$ and $\operatorname{WSM}_{\cos ^{1,2}}\left(\Upsilon_{1}, \Upsilon_{3}\right) \leq \operatorname{WSM}_{\cos ^{1,2}}\left(\Upsilon_{2}, \Upsilon_{3}\right)$

5.2. Cotangent Function Similarity Measure of q-RLDFSs. In this section, we use the cotangent function to develop a cotangent similarity measure (cotSM) for two $q$-RLDFNs $\Upsilon_{1}$ and $\Upsilon_{2}$. We also develop the weighted cotSM for two $q$ RLDFNs $\Upsilon_{1}$ and $\Upsilon_{2}$ and also prove some properties of the cot $\mathrm{SM}$ and WcotSM with distance measures. 
Definition 19. Consider two nonempty $q$-RLDFNs $\Upsilon_{1}=$ $\left(\left\langle u_{\Upsilon_{1}}\left(x_{i}\right), v_{\Upsilon_{1}}\left(x_{i}\right)\right\rangle,\left\langle a_{i}, b_{i}\right\rangle\right)$ and $\Upsilon_{2}=\left(\left\langle u_{\Upsilon_{2}}\left(x_{i}\right), v_{\Upsilon_{2}}\left(x_{i}\right)\right\rangle\right.$, $\left.\left\langle c_{i}, d_{i}\right\rangle\right)$ on the discrete nonempty set $\wp=\left(\rho_{1}, \rho_{2}, \ldots, \rho_{n}\right)$.
The SMs based on the cotangent function for two $q$-RLDFNs $\Upsilon_{1}$ and $\Upsilon_{2}$ are denoted by $\operatorname{SM}_{\text {cot }}\left(\Upsilon_{1}, \Upsilon_{2}\right)$ and defined by the following equation:

$$
\begin{aligned}
& \mathrm{SM}_{\cot ^{1}}\left(\Upsilon_{1}, \Upsilon_{2}\right)=\frac{1}{n} \sum_{i=1}^{n} \cot \left[\frac{\pi}{4}+\frac{\pi}{4}\left(\begin{array}{c}
\left|u_{\Upsilon_{1}}^{q}\left(x_{i}\right)-u_{\Upsilon_{2}}^{q}\left(x_{i}\right)\right| \\
\vee\left|v_{\Upsilon_{1}}^{q}\left(x_{i}\right)-v_{\Upsilon_{2}}^{q}\left(x_{i}\right)\right| \\
\vee\left|a_{i}^{q}-c_{i}^{q}\right| \vee\left|b_{i}^{q}-d_{i}^{q}\right|
\end{array}\right)\right], \\
& \mathrm{SM}_{\cot ^{2}}\left(\Upsilon_{1}, \Upsilon_{2}\right)=\frac{1}{n} \sum_{i=1}^{n} \cot \left[\frac{\pi}{4}+\frac{\pi}{4}\left(\begin{array}{c}
\left|u_{\Upsilon_{1}}^{q}\left(x_{i}\right)-u_{\Upsilon_{2}}^{q}\left(x_{i}\right)\right| \\
+\left|v_{\Upsilon_{1}}^{q}\left(x_{i}\right)-v_{\Upsilon_{2}}^{q}\left(x_{i}\right)\right| \\
+\left|a_{i}^{q}-c_{i}^{q}\right|+\left|b_{i}^{q}-d_{i}^{q}\right|
\end{array}\right)\right]
\end{aligned}
$$

Equations (46) and (48) satisfied the following properties:

(1) $0 \leq \operatorname{SM}_{\cot ^{1,2}}\left(\Upsilon_{1}, \Upsilon_{2}\right) \leq 1$

(2) $\mathrm{SM}_{\cot ^{1,2}}\left(\Upsilon_{1}, \Upsilon_{2}\right)=\operatorname{SM}_{\cot ^{1,2}}\left(\Upsilon_{2}, \Upsilon_{1}\right)$

(3) $\mathrm{SM}_{\cot ^{1,2}}\left(\Upsilon_{1}, \Upsilon_{2}\right)=1$ iff $\Upsilon_{1}=\Upsilon_{2}$

(4) If $\mathrm{SM}_{\cos 2}\left(\Upsilon_{1}, \Upsilon_{3}\right) \leq \mathrm{SM}_{1} \subseteq \Upsilon_{2} \subseteq \Upsilon_{3}, \Upsilon_{1}$ $\mathrm{SM}_{\cot , 2,2}\left(\Upsilon_{1}, \Upsilon_{3}\right) \leq \mathrm{SM}_{\cot , 1,2}\left(\Upsilon_{1}, \Upsilon_{2}\right)$

Proof.

(1) Since the cosine function values lie between the closed interval 0 and 1 and based on the cosine function, the $q$-RLDFN cosine similarity measures are also within $[0,1]$. Thus, the values of $\mathrm{SM}_{\text {cot }^{1,2}}\left(\Upsilon_{1}, \Upsilon_{2}\right)$ must lie between 0 and 1 .

(2) This is easily proven such that $\operatorname{SM}_{\cot ^{1,2}}\left(\Upsilon_{1}, \Upsilon_{2}\right)=$ $\mathrm{SM}_{\cot ^{1,2}}\left(\Upsilon_{2}, \Upsilon_{1}\right)$.

(3) This is easily proven such that $\operatorname{SM}_{\cot ^{1,2}}\left(\Upsilon_{1}, \Upsilon_{2}\right)=1$.

(4) Consider three $q$-RLDFNs, such as $\Upsilon_{1} \subseteq \Upsilon_{2} \subseteq \Upsilon_{3}$. Then,

$$
\begin{aligned}
u_{\Upsilon_{1}}^{q}\left(x_{i}\right) & \leq u_{\Upsilon_{2}}^{q}\left(x_{i}\right) \leq u_{\Upsilon_{3}}^{q}\left(x_{i}\right), \\
v_{\Upsilon_{1}}^{q}\left(x_{i}\right) & \geq v_{\Upsilon_{2}}^{q}\left(x_{i}\right) \geq v_{\Upsilon_{3}}^{q}\left(x_{i}\right), \\
a_{i}^{q} & \leq c_{i}^{q} \leq e_{i}^{q} \text { an } b_{i}^{q} \geq d_{i}^{q} \geq f_{i}^{q} .
\end{aligned}
$$

Then,

$$
\begin{aligned}
\left|u_{\Upsilon_{1}}^{q}\left(x_{i}\right)-u_{\Upsilon_{2}}^{q}\left(x_{i}\right)\right| & \leq\left|u_{\Upsilon_{1}}^{q}\left(x_{i}\right)-u_{\Upsilon_{2}}^{q}\left(x_{i}\right)\right|, \\
\left|u_{\Upsilon_{2}}^{q}\left(x_{i}\right)-u_{\Upsilon_{3}}^{q}\left(x_{i}\right)\right| & \leq\left|u_{\Upsilon_{1}}^{q}\left(x_{i}\right)-u_{\Upsilon_{3}}^{q}\left(x_{i}\right)\right|, \\
\left|v_{\Upsilon_{1}}^{q}\left(x_{i}\right)-v_{\Upsilon_{2}}^{q}\left(x_{i}\right)\right| & \leq\left|v_{\Upsilon_{1}}^{q}\left(x_{i}\right)-v_{\Upsilon_{2}}^{q}\left(x_{i}\right)\right|, \\
\left|v_{\Upsilon_{2}}^{q}\left(x_{i}\right)-v_{\Upsilon_{3}}^{q}\left(x_{i}\right)\right| & \leq\left|v_{\Upsilon_{1}}^{q}\left(x_{i}\right)-v_{\Upsilon_{3}}^{q}\left(x_{i}\right)\right|, \\
\left|a_{i}^{q}-c_{i}^{q}\right| & \leq\left|a_{i}^{q}-e_{i}^{q}\right| \text { an }\left|c_{i}^{q}-e_{i}^{q}\right| \leq\left|a_{i}^{q}-e_{i}^{q}\right|, \\
\left|b_{i}^{q}-d_{i}^{q}\right| & \leq\left|b_{i}^{q}-f_{i}^{q}\right| \text { an }\left|d_{i}^{q}-f_{i}^{q}\right| \leq\left|b_{i}^{q}-f_{i}^{q}\right| .
\end{aligned}
$$

We know that the cotangent function is a decreasing function in $[0, \pi / 4]$. Thus, $\operatorname{SM}_{\cot ^{1,2}}\left(\Upsilon_{1}, \Upsilon_{3}\right) \leq \operatorname{SM}_{\cot ^{1,2}}\left(\Upsilon_{1}, \Upsilon_{2}\right)$ and $\mathrm{SM}_{\cot ^{1,2}}\left(\Upsilon_{1}, \Upsilon_{3}\right) \leq \operatorname{SM}_{\cot ^{1,2}}\left(\Upsilon_{2}, \Upsilon_{3}\right)$.

Now, consider weight $w_{i}$ for each $x_{i}$, with the weight vector for all values of $x_{i}$. Then, we propose the weighted SM for two $q$-RLDFSs based on the cotangent function.

Definition 20. Consider two nonempty $q$-RLDFNs $\Upsilon_{1}=$ $\left(\left\langle u_{\Upsilon_{1}}\left(x_{i}\right), v_{\Upsilon_{1}}\left(x_{i}\right)\right\rangle,\left\langle a_{i}, b_{i}\right\rangle\right)$ and $\Upsilon_{2}=\left(\left\langle u_{\Upsilon_{2}}\left(x_{i}\right), v_{\Upsilon_{2}}\left(x_{i}\right)\right\rangle\right.$, $\left.\left\langle c_{i}, d_{i}\right\rangle\right)$ on the discrete nonempty set $\wp=\left(\rho_{1}, \rho_{2}, \ldots, \rho_{n}\right)$. Then, the weighted cosine similarity measure based on the cotangent function is denoted by $\operatorname{WSM}_{\cot ^{1,2}}\left(\Upsilon_{1}, \Upsilon_{2}\right)$ and given as

$$
\mathrm{WSM}_{\mathrm{cot}^{1}}\left(\Upsilon_{1}, \Upsilon_{2}\right)=\sum_{i=1}^{n} w_{i} \cot \left[\frac{\pi}{4}+\frac{\pi}{4}\left(\begin{array}{c}
\left|u_{\Upsilon_{1}}^{q}\left(x_{i}\right)-u_{\Upsilon_{2}}^{q}\left(x_{i}\right)\right| \\
\vee\left|v_{\Upsilon_{1}}^{q}\left(x_{i}\right)-v_{\Upsilon_{2}}^{q}\left(x_{i}\right)\right| \\
\vee\left|a_{i}^{q}-c_{i}^{q}\right| \vee\left|b_{i}^{q}-d_{i}^{q}\right|
\end{array}\right)\right],
$$




$$
\mathrm{WSM}_{\cot ^{2}}\left(\Upsilon_{1}, \Upsilon_{2}\right)=\sum_{i=1}^{n} w_{i} \cot \left[\frac{\pi}{4}+\frac{\pi}{4}\left(\begin{array}{c}
\left|u_{\Upsilon_{1}}^{q}\left(x_{i}\right)-u_{\Upsilon_{2}}^{q}\left(x_{i}\right)\right| \\
+\left|v_{\Upsilon_{1}}^{q}\left(x_{i}\right)-v_{\Upsilon_{2}}^{q}\left(x_{i}\right)\right| \\
+\left|a_{i}^{q}-c_{i}^{q}\right|+\left|b_{i}^{q}-d_{i}^{q}\right|
\end{array}\right)\right],
$$

where $w=\left(w_{1}, w_{2}, \ldots, w_{n}\right)^{T}$ is a weight vector of each $x_{i}$ such that $w_{i} \in[0,1]$ and $\sum_{i=1}^{n} w_{i}=1$. If we take $w_{i}=1 / n$, then equations (51) and (52) become equations (47) and (48). The WcotSM is a generalized form of cotSM. The WcotSM is also satisfied for two $q$-RLDFNs.

The WcotSM of three $q$-RLDFNs $\Upsilon_{1}, \Upsilon_{2}$, and $\Upsilon_{3}$ satisfied the following properties:

(1) $0 \leq \mathrm{WSM}_{\cot ^{1,2}}\left(\Upsilon_{1}, \Upsilon_{2}\right) \leq 1$

(2) $\operatorname{WSM}_{\cot ^{1,2}}\left(\Upsilon_{1}, \Upsilon_{2}\right)=\operatorname{WSM}_{\cot ^{1,2}}\left(\Upsilon_{2}, \Upsilon_{1}\right)$

(3) $\operatorname{WSM}_{\cot 1,2}\left(\Upsilon_{1}, \Upsilon_{2}\right)=1$ iff $\Upsilon_{1}=\Upsilon_{2}$

(4) If $\Upsilon_{1} \subseteq \Upsilon_{2} \subseteq \Upsilon_{3}$, then $\operatorname{WSM}_{\cot ^{1,2}}\left(\Upsilon_{1}, \Upsilon_{3}\right) \leq \mathrm{WSM}_{\text {cot }^{1,2}}$ $\left(\Upsilon_{1}, \Upsilon_{2}\right)$ and $\operatorname{WSM}_{\cot ^{1,2}}\left(\Upsilon_{1}, \Upsilon_{3}\right) \leq \operatorname{WSM}_{\cot ^{1,2}}\left(\Upsilon_{2}, \Upsilon_{3}\right)$

\section{Applications of Proposed Similarity Measures}

In the literature, some similarity measures of different fuzzy sets have been developed in different fields, i.e., decisionmaking, clustering analysis, data mining, pattern recognition, and supply chain management. This section provides the detailed description of applications of the proposed SM of the present paper. We are going to develop general multicriteria decision support systems for logistics and supply chain management. In case of logistics and provider selection, the detailed description of the algorithm is given in the following steps.

Let $A=\left(A_{1}, A_{2}, \ldots, A_{m}\right)$ be the collection of $m$ alternatives and $C=\left(C_{1}, C_{2}, \ldots, C_{n}\right)$ be the set of criteria. The criteria $C_{j}(j=1,2, \ldots, n)$ weight vector is $w=\left(w_{1}, w_{2}, \ldots\right.$, $\left.w_{n}\right)^{T}$, satisfying that $w_{j} \in[0,1]$ and $\sum_{j=1}^{n} w_{j}=1$, for $j=1,2, \ldots, n$. Suppose that $E=\left(E_{1}, E_{2}, \ldots, E_{p}\right)$ is the set of experts; every expert gives their assessment in the positive and negative grade to each criterion $C_{j}(j=1,2, \ldots, n)$ on the alternative $A_{i}(i=1,2, \ldots, m)$, respectively. So, we construct the decision matrix in the form of $q$-RLDFNs.

Then, the following steps are used to solve the MCDM problems, applying the cosine and cotangent similarity measures of $q$-RLDFNs.

Step 1: in this step, we construct a committee of the experts

Step 2: in this step, we collect information in the form of $q$-RLDFNs and make a decision matrix of the alternatives

Step 3: the proposed similarity measures are used to find the collective values of the decision matrix

Step 4: the alternatives are ranked according to the highest value of the alternatives, and the best one is chosen
6.1. Logistics Providers (LPs) and Supply Chain Management Problem. In this section, we have addressed a case study for selecting logistics providers (LPs). In our decision and ranking of alternatives, we use the proposed similarity measures. We connect our results with certain previous methods.

6.1.1. Case Study with the Numerical Approach. For the purpose of logistics support, the better selection of logistics providers (LPs) is now important to the growth and advancement of the supply chain theories. Over the past decades, there has been a drastic change in the course of decision support systems.

A supply chain is considered as a method with a full range of activities in which raw materials are converted into finally completed goods and then distributed by distribution, logistics, and retail to customers. As a supply chain management system, all interfunctional processes such as ordering, distribution, product delivery, and reverse logistics are taken into account [67-69].

Such systems allow experts in the supply chain to promote information flow, highly qualified decisions, and through internal coordination to increase the demand for goods and services [70]. In order to control the cost of the products in a textile factory, a DSS has aided decision makers in choosing effective ways to minimize overall cost of production.

Supply chain management can be considered sustainable when extended to all related aspects of the supply chain: energy conservation, final goods assurance, after-sales service, ethical job problems, reuse, and reverse logistics. In this way, arguing consumer expectations and then translating them into the supply chain considerations in this work may not only shift the supply chain towards a global and sustainable appearance but also strengthen the relevance of customer-based analysis. In the current group decision model, the task is to develop an alternative LP ranking based on the proposed similarity measures. The proposed method steps include a window for implementing costumer-driven weighting procedure for the criteria of decision. This factor allows for the decision process to satisfy external stakeholders and customers. We considered four decision makers to send us their opinions on the above logistics providers regarding the related factors for constructing a decision system. First, the necessary and the corresponding technical criteria for determining the logistics providers (LPs) of third parties are defined and implemented. The alternatives are true logistics and transport companies operating in France and Europe as a whole.

How to control the flow of our information? Where should our houses be located? What a framework should be 
introduced for transport and logistic? A managed and flexible supply chain is of paramount importance not only because of communication between all departments of the business (marketing, logistics, procurement, etc.) but also because of the extension of communication beyond its boundaries (suppliers, subcontractors, LPs, etc.).

Multiactivities of over 400 businesses, a network of almost 1500 members, ASLOG is now the leading French network of supply chain professionals. Its objectives include providing forward-looking goals, developing standards and qualifications, measuring and assessing logistic output, and eventually producing research distribution in collaboration with the benchmarking of best practices.

Step 1: Mathez, Bansard, Gefco, and Schneider are chosen to be four logistics provider companies, i.e., alternatives $A_{m}$, and examined by the suggested methods.

$A_{1}$ : Mathez group's main activities can be expanded from air, sea, and road freight, also packaging, supply and distribution management and optimizing, port agent, cargo, and cruise ship management. The main activities of the Mathez group can be expanded from air, sea, and road freight, packaging, deliver and distribution processing, port controller, and transport of goods.

$A_{2}$ : Bansard International offers a comprehensive platform for transport by sea, air, and road via agreements with leading airports to meet our clients' needs. Thus, Bansard International serves the main ports and airports. Moreover, this firm offers door-todoor support services to promote custom activities. They provide services such as air, marine, and road transport, logistics, and construction plants.

$A_{3}$ : Gefco offers effective logistics solutions worldwide for its industrial clients. The company is able to fulfil all criteria for the supply chain optimization process, land transport, distribution, shipment planning, and air flows.

$A_{4}$ : the company Schneider prepares and manages the transport of all kinds of goods between Europe and Switzerland. This company provides a type of daily activities for transport by air, sea, and road, reverse transportation, vegetables transportation, etc.

This case study focuses on applying the decision support model in the supply chain which aims to demonstrate how it can choose a third party LP as a partner from the pool of potential suppliers. The model's goal is to include the interests of consumers and stakeholders in the decision process so as to provide a global and detailed framework. Suppose the six technical factors denote technical requirements (decision criteria $C_{1}, C_{2}, \ldots, C_{6}$ ) which satisfy the four alternative LPs. On the contrary, the following criteria with weight vector $w=(0.11,0.31,0.15,0.17,0.14,0.12)^{T}$ are listed for LP evaluation as follows:

$$
\begin{aligned}
& C_{1} \text { : financial stability } \\
& C_{2} \text { : quality system }
\end{aligned}
$$

$C_{3}$ : delivery condition

$\mathrm{C}_{4}$ : services

$C_{5}$ : flexibility of the system

$\mathrm{C}_{6}$ : company stability

Step 1: in this step, we invite a team of experts to give their assessments

Step 2: now, every expert gives their assessments about the alternatives in the form of $q$-RLDFNs based on the six criteria given in Table 1

Step 3: now, we used the proposed similarity measures to find the collective values of the decision matrix, as given in Table 2

Based on the estimated results in Table 2, the degree of similarity between $A_{2}$ and $A$ is the highest, as determined by the other eight similarity measures. This indicates that all ten similarity measures think the alternative $A_{2}$ is closest to be the best alternative $A$ based on the principle of maximum similarity between $q$-rung linear Diophantine fuzzy sets ( $q$ RLDFSs). In other words, $A_{2}$ is the best alternative.

In Figure 1, we graphically show the ranking of the alternative given in Table 2 .

In practical DM problems, it is important to take the weights of elements into account if we take weights of criteria as $w=(0.11,0.31,0.15,0.17,0.14,0.12)^{T}$, respectively. Then, the weighted similarity measures proposed in this paper have been utilized to compute the similarity, and the results are listed in Table 3 (suppose $q=3$ ).

Based on the results in Table 3, the degree of similarity between $A_{2}$ and $A$ is the highest, as determined by the other eight similarity measures. This shows that, in all eight similarity measures, the alternative $A_{2}$ is closest to be the best alternative $A$ based on the principle of maximum similarity between $q$-RLDFNs. Thus, $A_{2}$ is the best alternative.

In Figure 2, we graphically show the ranking of the alternative given in Table 3 .

\section{Comparison and Discussion}

A comparison of our defined similarity measures with certain existing approaches in context $[29,40,52,53,62$, $63,71]$ has been done to illustrate the superiority of our defined similarity measures. Table 4 shows that the existing SMs are inaccessible when using $q$-ROF aggregation operators to solve the developed illustrated case of Section 6 . However, while the approaches described in $[65,66]$ contain Diophantine fuzzy information, they are inaccessible for solving the proposed information. Based on the study of Table 4, we can see that existing SMs lack rough information, and these approaches are unable to solve and rank the created case. As a result, the new method is more capable and effective than the existing approaches.

To demonstrate the effectiveness and scientific nature of our suggested approaches, we will compare them to the existing decision-making approaches such as [32-36, 57] with the $q$-rung orthopair fuzzy aggregation operators. The 
TABLE 1: The $q$-RLDFNs' information given by the expert.

\begin{tabular}{|c|c|c|c|c|c|c|}
\hline & $C_{1}$ & $C_{2}$ & $C_{3}$ & $C_{4}$ & $C_{5}$ & $C_{6}$ \\
\hline$A_{1}$ & $\left(\begin{array}{l}\langle 0.5,0.7\rangle \\
\langle 0.6,0.4\rangle\end{array}\right)$ & $\left(\begin{array}{l}\langle 0.8,0.3\rangle \\
\langle 0.7,0.4\rangle\end{array}\right)$ & $\left(\begin{array}{l}\langle 0.9,0.2\rangle \\
\langle 0.8,0.4\rangle\end{array}\right)$ & $\left(\begin{array}{l}\langle 0.6,0.7\rangle \\
\langle 0.3,0.6\rangle\end{array}\right)$ & $\left(\begin{array}{l}\langle 0.8,0.6\rangle \\
\langle 0.4,0.5\rangle\end{array}\right)$ & $\left(\begin{array}{l}\langle 0.5,0.7\rangle \\
\langle 0.6,0.4\rangle\end{array}\right)$ \\
\hline$A_{2}$ & $\left(\begin{array}{l}\langle 0.8,0.6\rangle \\
\langle 0.5,0.3\rangle\end{array}\right)$ & $\left(\begin{array}{l}\langle 0.9,0.7\rangle \\
\langle 0.2,0.6\rangle\end{array}\right)$ & $\left(\begin{array}{l}\langle 0.3,0.8\rangle \\
\langle 0.6,0.5\rangle\end{array}\right)$ & $\left(\begin{array}{l}\langle 0.8,0.6\rangle \\
\langle 0.5,0.3\rangle\end{array}\right)$ & $\left(\begin{array}{c}\langle 0.3,0.8\rangle \\
0.6,0.5\end{array}\right)$ & $\left(\begin{array}{l}\langle 0.9,0.4\rangle \\
\langle 0.5,0.3\rangle\end{array}\right)$ \\
\hline$A_{3}$ & $\left(\begin{array}{l}\langle 0.7,0.6\rangle \\
\langle 0.4,0.5\rangle\end{array}\right)$ & $\left(\begin{array}{l}\langle 0.9,0.1\rangle \\
\langle 0.8,0.3\rangle\end{array}\right)$ & $\left(\begin{array}{l}\langle 0.5,0.9\rangle \\
\langle 0.8,0.2\rangle\end{array}\right)$ & $\left(\begin{array}{l}\langle 0.5,0.9\rangle \\
\langle 0.8,0.2\rangle\end{array}\right)$ & $\left(\begin{array}{l}\langle 0.8,0.3\rangle \\
\langle 0.7,0.4\rangle\end{array}\right)$ & $\left(\begin{array}{l}\langle 0.8,0.7\rangle \\
\langle 0.2,0.6\rangle\end{array}\right)$ \\
\hline$A_{4}$ & $\left(\begin{array}{l}\langle 0.9,0.4\rangle \\
\langle 0.6,0.3\rangle\end{array}\right)$ & $\left(\begin{array}{l}\langle 0.5,0.6\rangle \\
\langle 0.3,0.9\rangle\end{array}\right)$ & $\left(\begin{array}{l}\langle 0.6,0.7\rangle \\
\langle 0.3,0.6\rangle\end{array}\right)$ & $\left(\begin{array}{l}\langle 0.9,0.2\rangle \\
\langle 0.7,0.4\rangle\end{array}\right)$ & $\left(\begin{array}{l}\langle 0.5,0.6\rangle \\
\langle 0.3,0.9\rangle\end{array}\right)$ & $\left(\begin{array}{l}\langle 0.9,0.1\rangle \\
\langle 0.8,0.3\rangle\end{array}\right)$ \\
\hline$A$ & $\left(\begin{array}{l}\langle 0.8,0.5\rangle \\
\langle 0.7,0.2\rangle\end{array}\right)$ & $\left(\begin{array}{l}\langle 0.4,0.8\rangle \\
\langle 0.6,0.5\rangle\end{array}\right)$ & $\left(\begin{array}{l}\langle 0.5,0.8\rangle \\
\langle 0.7,0.4\rangle\end{array}\right)$ & $\left(\begin{array}{l}\langle 0.4,0.7\rangle \\
\langle 0.6,0.3\rangle\end{array}\right)$ & $\left(\begin{array}{l}\langle 0.6,0.5\rangle \\
\langle 0.7,0.4\rangle\end{array}\right)$ & $\left(\begin{array}{c}\langle 0.7,0.2\rangle \\
\langle 0.4,0.6\rangle\end{array}\right)$ \\
\hline
\end{tabular}

TABLe 2: The similarity measures between $A_{i}$ an $\mathrm{d} A$.

\begin{tabular}{lccc}
\hline Similarity measure & $\left(A_{1}, A\right)$ & $\left(A_{2}, A\right)$ & $\left(A_{3}, A\right)$ \\
\hline $\left.\operatorname{JSM}_{(}, A\right)$ & 0.378 & 0.580 & 0.601 \\
$\operatorname{ESM}_{\mathrm{NHD}}\left(A_{i}, A\right)$ & 0.823 & 0.864 & 0.841 \\
$\operatorname{ESM}_{\mathrm{NED}}\left(A_{i}, A\right)$ & 0.766 & 0.793 & 0.781 \\
$\mathrm{CSM}\left(A_{i}, A\right)$ & 0.552 & 0.746 & 0.811 \\
$\operatorname{SM}_{\cos ^{1}}\left(A_{i}, A\right)$ & 0.683 & 0.790 & 0.612 \\
$\operatorname{SM}_{\cos ^{2}}\left(A_{i}, A\right)$ & 0.734 & 0.848 & 0.651 \\
$\operatorname{SM}_{\cot ^{1}}\left(A_{i}, A\right)$ & 0.572 & 0.624 & 0.532 \\
$\operatorname{SM}_{\cot ^{2}}\left(A_{i}, A\right)$ & 0.614 & 0.672 & 0.586 \\
\hline
\end{tabular}

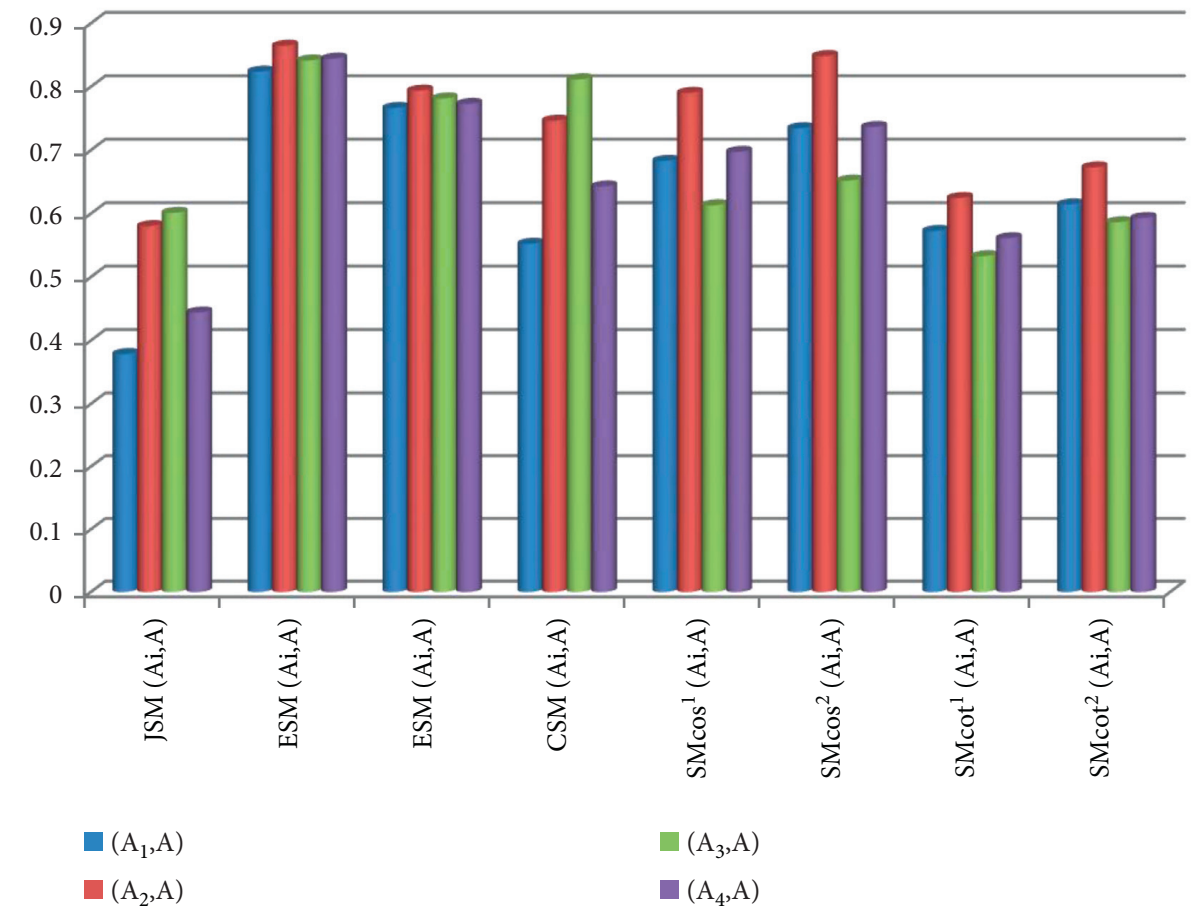

Figure 1: The ranking of the alternative given in Table 2. 
TABLE 3: The weighted similarity measures between $A_{i}$ an $\mathrm{d} A$.

\begin{tabular}{|c|c|c|c|c|}
\hline Similarity measure & $\left(A_{1}, A\right)$ & $\left(A_{2}, A\right)$ & $\left(A_{3}, A\right)$ & $\left(A_{4}, A\right)$ \\
\hline $\operatorname{WJSM}\left(A_{i}, A\right)$ & 0.359 & 0.529 & 0.528 & 0.423 \\
\hline $\operatorname{WESM}_{\mathrm{NHD}}\left(A_{i}, A\right)$ & 0.964 & 0.969 & 0.964 & 0.970 \\
\hline $\operatorname{WESM}_{\mathrm{NED}}\left(A_{i}, A\right)$ & 0.892 & 0.900 & 0.886 & 0.895 \\
\hline $\operatorname{WCSM}\left(A_{i}, A\right)$ & 0.522 & 0.797 & 0.733 & 0.622 \\
\hline $\mathrm{WSM}_{\cos ^{1}}\left(A_{i}, A\right)$ & 0.721 & 0.793 & 0.772 & 0.668 \\
\hline $\operatorname{WSM}_{\cos ^{2}}\left(A_{i}, A\right)$ & 0.761 & 0.849 & 0.813 & 0.709 \\
\hline $\mathrm{WSM}_{\cot ^{1}}\left(A_{i}, A\right)$ & 0.535 & 0.582 & 0.562 & 0.486 \\
\hline $\operatorname{WSM}_{\cot ^{2}}\left(A_{i}, A\right)$ & 0.580 & 0.635 & 0.599 & 0.540 \\
\hline
\end{tabular}

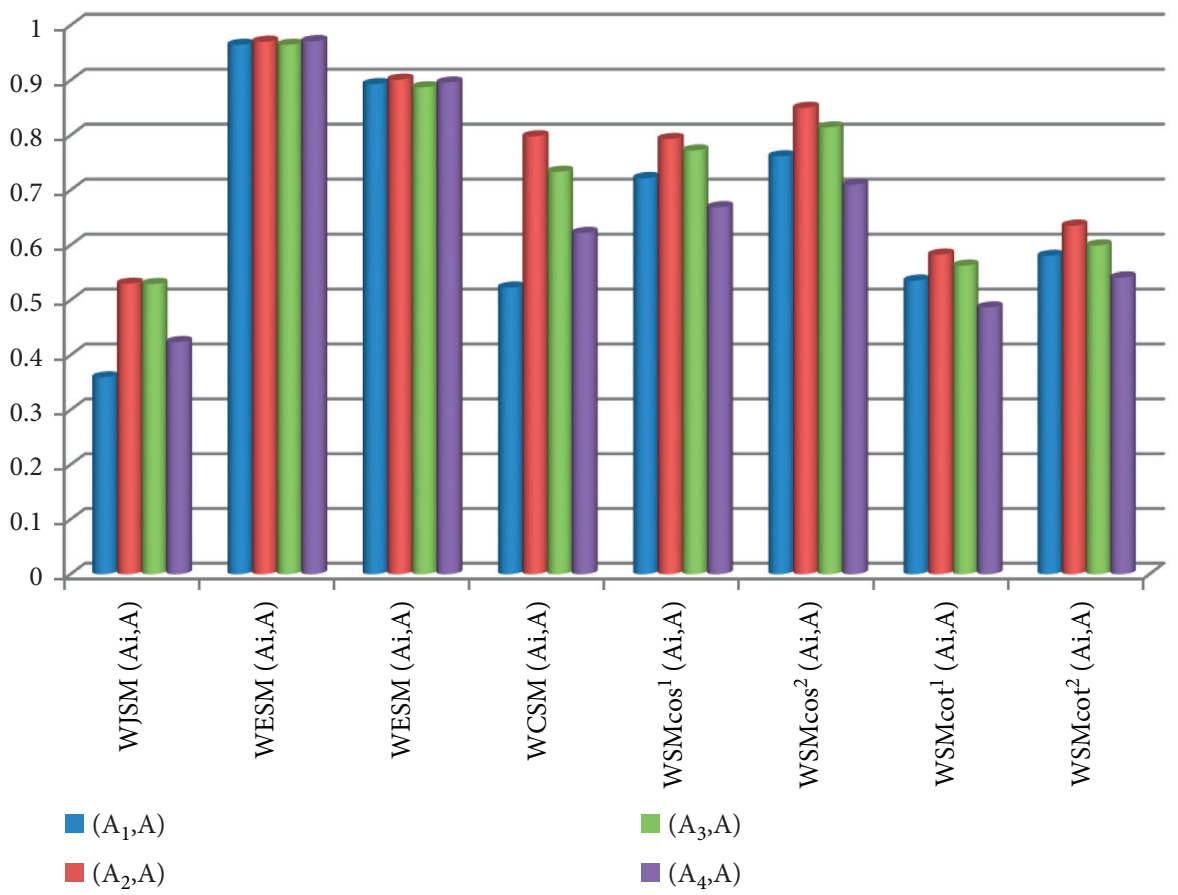

FIgURE 2: The ranking of the alternative given in Table 3.

TABle 4: Different methods and their ranking.

\begin{tabular}{lcc}
\hline Methods & Appraisal scores of alternatives & Ranking \\
\hline Du [29] & Inaccessible & $\times$ \\
Peng and Liu [63] & Inaccessible & $\times$ \\
Son and Phong [52] & Inaccessible & $\times$ \\
Wu et al. [71] & Inaccessible & $\times$ \\
Wei and Wei [53] & Inaccessible & $\times$ \\
Wang et al. [62] & Inaccessible & $\times$ \\
Ye [40] & Inaccessible & $\times$ \\
Methods & Remarks & Parameterization \\
$q$-RLDFS [66] & $q-\operatorname{RLDFS}$ covers this situation, \\
& $0 \leq(a)^{q} \mu_{D(x)}+(b)^{q} v_{D(x)} \leq 1$, & Yes \\
LDFS [65] & $\mathrm{LDFS} \operatorname{covers}$ this $\operatorname{situation,}$ & No \\
\end{tabular}

aggregated results of a comparative examination of existing methods using our example are listed in Table 5 with criteria weight vector $w=(0.11,0.31,0.15,0.17,0.14,0.12)^{T}$.
To date, we can see that cosine and cotangent SMs with $q$ ROFSs have been examined by a large number of researchers. However, as previously indicated, some particular scenarios 
TABLE 5: Score values and ranking order of the existing methods.

\begin{tabular}{lccccc}
\hline & $S\left(A_{1}\right)$ & $S\left(A_{2}\right)$ & $S\left(A_{3}\right)$ & $S\left(A_{4}\right)$ & Ranking \\
\hline $\begin{array}{l}\text { Liu and Wang } \\
\text { [32] }\end{array}$ & 0.692 & 0.743 & 0.729 & 0.663 & $A_{2}>A_{3}>A_{1}>A_{4}$ \\
Liu and Liu & 0.596 & 0.679 & 0.668 & 0.579 & $A_{2}>A_{3}>A_{1}>A_{4}$ \\
[36] & & & & & $A_{2}>A_{3}>A_{1}>A_{4}$ \\
Wei et al. [34] & 0.532 & 0.573 & 0.554 & 0.509 & $A_{1}$ \\
Wei et al. [33] & 0.822 & 0.885 & 0.837 & 0.793 & $A_{2}>A_{3}>A_{1}>A_{4}$ \\
Yang and Pang & 0.233 & 0.294 & 0.276 & 0.247 & $A_{2}>A_{3}>A_{4}>A_{1}$ \\
[35] & & 0.480 & 0.458 & 0.413 & $A_{2}>A_{3}>A_{1}>A_{4}$ \\
Zeng et al. [57] & 0.442 & 0.45
\end{tabular}

are not covered by $q$-ROFSs. As a result, algorithms based on SMs with a $q$-ROFS are incapable of dealing with such problems. The cosine and cotangent similarity measures with $q$-ROF information are special cases of the SMs described in this study with $q$-rung linear Diophantine fuzzy information. Because of the $q$ th power of reference parameters, this notion is impressive in that it covers the valuation spaces of IFSs, PyFSs, $q$-ROFSs, and LDFSs. As a result, our specified SMs are better suited and generalized to cope with the real-world situation than the current ones.

\section{Conclusion}

Based on $q$-rung linear Diophantine fuzzy sets ( $q$-RLDFSs), we proposed another form of similarity measures by considering the function of membership degree (MD), nonmembership degree (NMD), and reference parameters (RPs) in $q$-RLDFSs. The $q$-RLDFS model is more effective and versatile than other techniques due to the use of RPs. In addition, for the $q$-RLDFSs, we presented a family of similarity measures such as Jaccard SMs, exponential SMs, and cosine and cotangent SMs between $q$-RLDFSs by considering MD, NMD, and RPs. The concept of the qth power of reference parameters will provide a more versatile and efficient basis for fuzzy system modeling and decision-making under uncertainty, covering the space of existing structures as well as the space of MD, NMD, and RPs. It is important to note that if we increase the value of $q$, the Diophantine space expands, giving the large boundary limits to express a wider range of fuzzy data. We implemented the proposed MCDM methods under the guidance of a case study for the selection of the best logistics provider. We conclude that the current DM method is appropriate and stable and can be effectively implemented for multicriteria group decision-making problems.

In the future, study on $q$-RLDFSs could focus on various types of decision-making challenges, such as staff selection, investment, machine selection, project selection, and production systems.

\section{Data Availability}

No data were used to support this study.

\section{Ethical Approval}

This article does not contain any studies with human participants or animals performed by any of the authors.

\section{Conflicts of Interest}

The authors declare that they have no conflicts of interest.

\section{Acknowledgments}

The authors would like to thank the Deanship of Scientific Research at Umm Al-Qura University for supporting this work through grant no. 19-SCI-1-01-0041.

\section{References}

[1] P. Zaraté, "Cooperative decision-making," Tools for Collaborative Decision-Making, John Wiley \& Sons, Hoboken, NJ, USA, pp. 31-38, 2013.

[2] M. Yazdani, P. Zarate, A. Coulibaly, and E. K. Zavadskas, “A group decision making support system in logistics and supply chain management," Expert Systems with Applications, vol. 88, pp. 376-392, 2017.

[3] Z. X. Guo, E. W. T. Ngai, C. Yang, and X. Liang, "An RFIDbased intelligent decision support system architecture for production monitoring and scheduling in a distributed manufacturing environment," International Journal of Production Economics, vol. 159, pp. 16-28, 2015.

[4] X. F. Zha, R. D. Sriram, M. G. Fernandez, and F. Mistree, "Knowledge-intensive collaborative decision support for design processes: a hybrid decision support model and agent," Computers in Industry, vol. 59, no. 9, pp. 905-922, 2008.

[5] Y. Kristianto, A. Gunasekaran, P. Helo, and M. Sandhu, "A decision support system for integrating manufacturing and product design into the reconfiguration of the supply chain networks," Decision Support Systems, vol. 52, no. 4, pp. 790-801, 2012.

[6] J. Scott, W. Ho, P. K. Dey, and S. Talluri, "A decision support system for supplier selection and order allocation in stochastic, multi-stakeholder and multi-criteria environments," International Journal of Production Economics, vol. 166, pp. 226-237, 2015.

[7] P. Shi, B. Yan, S. Shi, and C. Ke, "A decision support system to select suppliers for a sustainable supply chain based on a systematic DEA approach," Information Technology and Management, vol. 16, no. 1, pp. 39-49, 2015.

[8] L. Zedah, "Fuzzy sets," Information and Control, vol. 8, pp. 338-353, 1965.

[9] K. T. Atanassov, "Intuitionistic fuzzy sets," Fuzzy Sets and Systems, vol. 20, no. 1, pp. 87-96, 1986.

[10] R. R. Yager, "Pythagorean membership grades in multicriteria decision making," IEEE Transactions on Fuzzy Systems, vol. 22, no. 4, pp. 958-965, 2013.

[11] R. X. Nie, Z. P. Tian, J. Q. Wang, and J. H. Hu, "Pythagorean fuzzy multiple criteria decision analysis based on Shapley fuzzy measures and partitioned normalized weighted Bonferroni mean operator," International Journal of Intelligent Systems, vol. 34, no. 2, pp. 297-324, 2019.

[12] W. Yang, J. Shi, Y. Liu, Y. Pang, and R. Lin, "Pythagorean fuzzy interaction partitioned Bonferroni mean operators and their application in multiple-attribute decision-making," Complexity, vol. 2018, Article ID 3606245, 13 pages, 2018.

[13] C. Zhang, D. Li, Y. Mu, and D. Song, "A Pythagorean fuzzy multigranulation probabilistic model for mine ventilator fault diagnosis," Complexity, vol. 2018, Article ID 7125931, 22 pages, 2018.

[14] L. Zhu, X. Liang, L. Wang, and X. Wu, "Generalized pythagorean fuzzy point operators and their application in 
multi-attributes decision making," Journal of Intelligent and Fuzzy Systems, vol. 35, no. 2, pp. 1407-1418, 2018.

[15] C. Zhao, X. Tang, and L. Yuan, "MAGDM method with pythagorean 2-tuple linguistic information and applications in the HSE performance assessment of laboratory," Mathematical Problems in Engineering, vol. 2018, Article ID 3732808, 9 pages, 2018.

[16] R. Verma, "On intuitionistic fuzzy order divergence and entropy measures with MABAC method for multiple attribute group decision-making," Journal of Intelligent and Fuzzy Systems, pp. 1-27, 2021, Preprint).

[17] X. Zhang and Z. Xu, "Extension of TOPSIS to multiple criteria decision making with Pythagorean fuzzy sets," International Journal of Intelligent Systems, vol. 29, no. 12, pp. 1061-1078, 2014.

[18] X. Peng and Y. Yang, "Some results for Pythagorean fuzzy sets," International Journal of Intelligent Systems, vol. 30, no. 11, pp. 1133-1160, 2015.

[19] M. Z. Reformat and R. R. Yager, "Suggesting recommendations using pythagorean fuzzy sets illustrated using netflix movie data," Information Processing and Management of Uncertainty in Knowledge-Based Systems, Springer, Cham, Switzerland, pp. 546-556, 2014.

[20] H. Garg, "A new generalized Pythagorean fuzzy information aggregation using Einstein operations and its application to decision making," International Journal of Intelligent Systems, vol. 31, no. 9, pp. 886-920, 2016.

[21] P. Ren, Z. Xu, and X. Gou, "Pythagorean fuzzy TODIM approach to multi-criteria decision making," Applied Soft Computing, vol. 42, pp. 246-259, 2016.

[22] G. Wei and M. Lu, "Pythagorean fuzzy Maclaurin symmetric mean operators in multiple attribute decision making," International Journal of Intelligent Systems, vol. 33, no. 5, pp. 1043-1070, 2018.

[23] S. Zeng, J. Chen, and X. Li, "A hybrid method for Pythagorean fuzzy multiple-criteria decision making," International Journal of Information Technology and Decision Making, vol. 15, no. 2, pp. 403-422, 2016.

[24] S. M. Chen and C. H. Chang, "A new similarity measure between intuitionistic fuzzy sets based on transformation techniques,"vol. 1, pp. 396-402, in Proceedings of the 2014 International Conference on Machine Learning and Cybernetics, vol. 1, pp. 396-402, IEEE, Lanzhou, China, 2014 July.

[25] D. Liang, Z. Xu, and A. P. Darko, "Projection model for fusing the information of Pythagorean fuzzy multicriteria group decision making based on geometric Bonferroni mean," International Journal of Intelligent Systems, vol. 32, no. 9, pp. 966-987, 2017.

[26] D. Liang, Y. Zhang, Z. Xu, and A. P. Darko, "Pythagorean fuzzy Bonferroni mean aggregation operator and its accelerative calculating algorithm with the multithreading," International Journal of Intelligent Systems, vol. 33, no. 3, pp. 615-633, 2018.

[27] X. Peng, H. Yuan, and Y. Yang, "Pythagorean fuzzy information measures and their applications," International Journal of Intelligent Systems, vol. 32, no. 10, pp. 991-1029, 2017.

[28] G. Wei and M. Lu, "Dual hesitant Pythagorean fuzzy Hamacher aggregation operators in multiple attribute decision making," Archives of Control Sciences, vol. 27, 2017.

[29] W. S. Du, "Minkowski-type distance measures for generalized orthopair fuzzy sets," International Journal of Intelligent Systems, vol. 33, no. 4, pp. 802-817, 2018.
[30] P. A. Ejegwa, "Modified Zhang and Xu's distance measure for Pythagorean fuzzy sets and its application to pattern recognition problems," Neural Computing \& Applications, vol. 32, no. 14, pp. 10199-10208, 2020.

[31] R. R. Yager, "Generalized orthopair fuzzy sets," IEEE Transactions on Fuzzy Systems, vol. 25, no. 5, pp. 1222-1230, 2016.

[32] P. Liu and P. Wang, "Some q-rung orthopair fuzzy aggregation operators and their applications to multiple-attribute decision making," International Journal of Intelligent Systems, vol. 33, no. 2, pp. 259-280, 2018.

[33] G. Wei, C. Wei, J. Wang, H. Gao, and Y. Wei, "Some q-rung orthopair fuzzy maclaurin symmetric mean operators and their applications to potential evaluation of emerging technology commercialization," International Journal of Intelligent Systems, vol. 34, no. 1, pp. 50-81, 2019.

[34] G. Wei, H. Gao, and Y. Wei, "Some q-rung orthopair fuzzy Heronian mean operators in multiple attribute decision making," International Journal of Intelligent Systems, vol. 33, no. 7, pp. 1426-1458, 2018.

[35] W. Yang and Y. Pang, "New q-rung orthopair fuzzy partitioned Bonferroni mean operators and their application in multiple attribute decision making," International Journal of Intelligent Systems, vol. 34, no. 3, pp. 439-476, 2019.

[36] P. Liu and W. Liu, "Multiple-attribute group decision-making based on power Bonferroni operators of linguisticq-rung orthopair fuzzy numbers," International Journal of Intelligent Systems, vol. 34, no. 4, pp. 652-689, 2019.

[37] Q. Lei and Z. Xu, "Relationships between two types of intuitionistic fuzzy definite integrals," IEEE Transactions on Fuzzy Systems, vol. 24, no. 6, pp. 1410-1425, 2016.

[38] L. Dengfeng and C. Chuntian, "New similarity measures of intuitionistic fuzzy sets and application to pattern recognitions," Pattern Recognition Letters, vol. 23, no. 1-3, pp. 221-225, 2002.

[39] J. Ye, Z. Fang, and W. Cui, "Vector similarity measures of Q-linguistic neutrosophic variable sets and their multi-attribute decision making method," Symmetry, vol. 10, no. 10, p. 531, 2018.

[40] J. Ye, "Multiple-attribute decision-making method using similarity measures of single-valued neutrosophic hesitant fuzzy sets based on least common multiple cardinality," Journal of Intelligent and Fuzzy Systems, vol. 34, no. 6, pp. 4203-4211, 2018.

[41] W. Zeng, D. Li, and Q. Yin, "Distance and similarity measures of Pythagorean fuzzy sets and their applications to multiple criteria group decision making," International Journal of Intelligent Systems, vol. 33, no. 11, pp. 2236-2254, 2018.

[42] H. B. Mitchell, "On the Dengfeng-Chuntian similarity measure and its application to pattern recognition," Pattern Recognition Letters, vol. 24, no. 16, pp. 3101-3104, 2003.

[43] J. S. Park, Y. C. Kwun, and J. H. Park, "Some results and example for compatible maps of type $(\beta)$ on intuitionistic fuzzy metric space," in Fuzzy Information and Engineering, vol. 2, pp. 629-638, Springer, Berlin, Heidelberg, 2009.

[44] V. Torra and Y. Narukawa, "On hesitant fuzzy sets and decision," in Proceedings of the 2009 IEEE International Conference on Fuzzy Systems, pp. 1378-1382, IEEE, Jeju Island, Korea, August 2009.

[45] M. Xia and Z. Xu, "Some new similarity measures for intuitionistic fuzzy values and their application in group decision making," Journal of Systems Science and Systems Engineering, vol. 19, no. 4, pp. 430-452, 2010. 
[46] J. Ye, "Cosine similarity measures for intuitionistic fuzzy sets and their applications," Mathematical and Computer Modelling, vol. 53, no. 1-2, pp. 91-97, 2011.

[47] K.-C. Hung, "Applications of medical information: using an enhanced likelihood measured approach based on intuitionistic fuzzy sets," IIE Transactions on Healthcare Systems Engineering, vol. 2, no. 3, pp. 224-231, 2012.

[48] L. L. Shi and J. Ye, "Study on fault diagnosis of turbine using an improved cosine similarity measure for vague sets," Journal of Applied Sciences, vol. 13, no. 10, pp. 1781-1786, 2013.

[49] T. Maoying, "A new fuzzy similarity measure based on cotangent function for medical diagnosis," Adv. Model. Optim, vol. 15, no. 2, pp. 151-156, 2013.

[50] P. Rajarajeswari and N. Uma, "Intuitionistic fuzzy multi similarity measure based on cotangent function," Int. Journal of Eng. Research and Tech, vol. 2, no. 11, pp. 1323-1329, 2013.

[51] E. Szmidt, Distances and Similarities in Intuitionistic Fuzzy Sets, Vol. 307, Springer International Publishing, Cham, Switzerland, 2014.

[52] L. H. Son and P. H. Phong, "On the performance evaluation of intuitionistic vector similarity measures for medical diagnosis1," Journal of Intelligent and Fuzzy Systems, vol. 31, no. 3, pp. 1597-1608, 2016.

[53] G. Wei and Y. Wei, "Similarity measures of Pythagorean fuzzy sets based on the cosine function and their applications," International Journal of Intelligent Systems, vol. 33, no. 3, pp. 634-652, 2018.

[54] J. Ye, "Similarity measures of intuitionistic fuzzy sets based on cosine function for the decision making of mechanical design schemes," Journal of Intelligent and Fuzzy Systems, vol. 30, no. 1, pp. 151-158, 2016.

[55] Z. Li and M. Lu, "Some novel similarity and distance measures of pythagorean fuzzy sets and their applications," Journal of Intelligent and Fuzzy Systems, vol. 37, no. 2, pp. 1781-1799, 2019.

[56] R. Verma and J. M. Merigó, "On generalized similarity measures for Pythagorean fuzzy sets and their applications to multiple attribute decision-making," International Journal of Intelligent Systems, vol. 34, no. 10, pp. 2556-2583, 2019.

[57] S. Zeng, Y. Hu, and X. Xie, "Q-rung orthopair fuzzy weighted induced logarithmic distance measures and their application in multiple attribute decision making," Engineering Applications of Artificial Intelligence, vol. 100, Article ID 104167, 2021.

[58] M. Akram, G. Ali, M. A. Butt, and J. C. R. Alcantud, "Novel MCGDM analysis under m-polar fuzzy soft expert sets," Neural Computing \& Applications, vol. 33, no. 1, pp. 1-21, 2021.

[59] M. Akram, A. Ghous, and J. C. R. Alcantud, "Attributes reduction algorithms for m-polar fuzzy relation decision systems," International Journal of Approximate Reasoning, vol. 140, 2021.

[60] G. Ali and M. Sarwar, "Novel technique for group decisionmaking under fuzzy parameterized-rung orthopair fuzzy soft expert framework," Mathematical Problems in Engineering, vol. 2021, Article ID 5449403, 22 pages, 2021.

[61] G. Ali and M. Akram, "Decision-making method based on fuzzy N-soft expert sets," Arabian Journal for Science and Engineering, vol. 45, no. 12, pp. 10381-10400, 2020.

[62] P. Wang, J. Wang, G. Wei, and C. Wei, "Similarity measures of q-rung orthopair fuzzy sets based on cosine function and their applications," Mathematics, vol. 7, no. 4, p. 340, 2019.
[63] X. Peng and L. Liu, "Information measures for q -rung orthopair fuzzy sets," International Journal of Intelligent Systems, vol. 34, no. 8, pp. 1795-1834, 2019.

[64] M. I. Ali, "Another view on q-rung orthopair fuzzy sets," International Journal of Intelligent Systems, vol. 33, no. 11, pp. 2139-2153, 2018.

[65] M. Riaz and M. R. Hashmi, "Linear Diophantine fuzzy set and its applications towards multi-attribute decision-making problems," Journal of Intelligent and Fuzzy Systems, vol. 37, no. 4, pp. 5417-5439, 2019.

[66] A. O. Almagrabi, S. Abdullah, M. Shams, Y. D. Al-Otaibi, and S. Ashraf, "A new approach to q-linear Diophantine fuzzy emergency decision support system for COVID19," Journal of Ambient Intelligence and Humanized Computing, pp. 1-27, 2021, inpress.

[67] M. Brandenburg, K. Govindan, J. Sarkis, and S. Seuring, "Quantitative models for sustainable supply chain management: developments and directions," European Journal of Operational Research, vol. 233, no. 2, pp. 299-312, 2014.

[68] B. Fahimnia, J. Sarkis, and H. Davarzani, "Green supply chain management: a review and bibliometric analysis," International Journal of Production Economics, vol. 162, pp. 101-114, 2015.

[69] M. Yazdani, S. Hashemkhani Zolfani, and E. K. Zavadskas, "New integration of MCDM methods and QFD in the selection of green suppliers," Journal of Business Economics and Management, vol. 17, no. 6, pp. 1097-1113, 2016.

[70] C. Chandra and S. Kumar, "An application of a system analysis methodology to manage logistics in a textile supply chain," Supply Chain Management: An International Journal, vol. 5, no. 5, 2000.

[71] H. Wu, Y. Yuan, L. Wei, and L. Pei, “On entropy, similarity measure and cross-entropy of single-valued neutrosophic sets and their application in multi-attribute decision making," Soft Computing, vol. 22, no. 22, pp. 7367-7376, 2018. 